\title{
Some Aspects of Hankel Matrices in Coding Theory and Combinatorics
}

\author{
Ulrich Tamm \\ Department of Computer Science \\ University of Chemnitz \\ 09107 Chemnitz, Germany \\ tamm@informatik.tu-chemnitz.de
}

Submitted: December 8, 2000; Accepted: May 26, 2001.

MR Subject Classifications: primary 05A15, secondary 15A15, 94B35

\begin{abstract}
Hankel matrices consisting of Catalan numbers have been analyzed by various authors. DesainteCatherine and Viennot found their determinant to be $\prod_{1 \leq i \leq j \leq k} \frac{i+j+2 n}{i+j}$ and related them to the Bender - Knuth conjecture. The similar determinant formula $\prod_{1 \leq i \leq j \leq k} \frac{i+j-1+2 n}{i+j-1}$ can be shown to hold for Hankel matrices whose entries are successive middle binomial coefficients $\left(\begin{array}{c}2 m+1 \\ m\end{array}\right)$. Generalizing the Catalan numbers in a different direction, it can be shown that determinants of Hankel matrices consisting of numbers $\frac{1}{3 m+1}\left(\begin{array}{c}3 m+1 \\ m\end{array}\right)$ yield an alternate expression of two Mills Robbins - Rumsey determinants important in the enumeration of plane partitions and alternating sign matrices. Hankel matrices with determinant 1 were studied by Aigner in the definition of Catalan - like numbers. The well - known relation of Hankel matrices to orthogonal polynomials further yields a combinatorial application of the famous Berlekamp - Massey algorithm in Coding Theory, which can be applied in order to calculate the coefficients in the three - term recurrence of the family of orthogonal polynomials related to the sequence of Hankel matrices.
\end{abstract}

\section{Introduction}

A Hankel matrix (or persymmetric matrix)

$$
A_{n}=\left(\begin{array}{ccccc}
c_{0} & c_{1} & c_{2} & \ldots & c_{n-1} \\
c_{1} & c_{2} & c_{3} & \ldots & c_{n} \\
c_{2} & c_{3} & c_{4} & \ldots & c_{n+1} \\
\vdots & \vdots & \vdots & & \vdots \\
c_{n-1} & c_{n} & c_{n+1} & \ldots & c_{2 n-2}
\end{array}\right)
$$

is a matrix $\left(a_{i j}\right)$ in which for every $r$ the entries on the diagonal $i+j=r$ are the same, i.e., $a_{i, r-i}=c_{r}$ for some $c_{r}$. 
For a sequence $c_{0}, c_{1}, c_{2}, \ldots$ of real numbers we also consider the collection of Hankel matrices $A_{n}^{(k)}, k=0,1, \ldots, n=1,2, \ldots$, where

$$
A_{n}^{(k)}=\left(\begin{array}{ccccc}
c_{k} & c_{k+1} & c_{k+2} & \ldots & c_{k+n-1} \\
c_{k+1} & c_{k+2} & c_{k+3} & \ldots & c_{k+n} \\
c_{k+2} & c_{k+3} & c_{k+4} & \ldots & c_{k+n+1} \\
\vdots & \vdots & \vdots & & \vdots \\
c_{k+n-1} & c_{k+n} & c_{k+n+1} & \ldots & c_{k+2 n-2}
\end{array}\right) .
$$

So the parameter $n$ denotes the size of the matrix and the $2 n-1$ successive elements $c_{k}, c_{k+1}, \ldots, c_{k+2 n-2}$ occur in the diagonals of the Hankel matrix.

We shall further denote the determinant of a Hankel matrix (1.2) by

$$
d_{n}^{(k)}=\operatorname{det}\left(A_{n}^{(k)}\right)
$$

Hankel matrices have important applications, for instance, in the theory of moments, and in Padé approximation. In Coding Theory, they occur in the Berlekamp - Massey algorithm for the decoding of $\mathrm{BCH}$ - codes. Their connection to orthogonal polynomials often yields useful applications in Combinatorics: as shown by Viennot [76] Hankel determinants enumerate certain families of weighted paths, Catalan - like numbers as defined by Aigner [2] via Hankel determinants often yield sequences important in combinatorial enumeration, and as a recent application, they turned out to be an important tool in the proof of the refined alternating sign matrix conjecture.

The framework for studying combinatorial applications of Hankel matrices and further aspects of orthogonal polynomials was set up by Viennot [76]. Of special interest are determinants of Hankel matrices consisting of Catalan numbers $\frac{1}{2 m+1}\left(\begin{array}{c}2 m+1 \\ m\end{array}\right)$. Desainte Catherine and Viennot [24] provided a formula for $\operatorname{det}\left(A_{n}^{(k)}\right)$ and all $n \geq 1, k \geq 0$ in case that the entries $c_{m}$ are Catalan numbers, namely

For the sequence $c_{m}=\frac{1}{2 m+1}\left(\begin{array}{c}2 m+1 \\ m\end{array}\right), m=0,1, \ldots$ of Catalan numbers it is

$$
d_{n}^{(0)}=d_{n}^{(1)}=1, \quad d_{n}^{(k)}=\prod_{1 \leq i \leq j \leq k-1} \frac{i+j+2 n}{i+j} \quad \text { for } k \geq 2, n \geq 1 .
$$

Desainte-Catherine and Viennot [24] also gave a combinatorial interpretation of this determinant in terms of special disjoint lattice paths and applications to the enumeration of Young tableaux, matchings, etc..

They studied (1.4) as a companion formula for $\prod_{1 \leq i \leq j \leq k} \frac{i+j-1+c}{i+j-1}$, which for integer $c$ was shown by Gordon (cf. [67]) to be the generating function for certain Young tableaux.

For even $c=2 n$ this latter formula also can be expressed as a Hankel determinant formed of successive binomial coefficients $\left(\begin{array}{c}2 m+1 \\ m\end{array}\right)$.

For the binomial coefficients $c_{m}=\left(\begin{array}{c}2 m+1 \\ m\end{array}\right), m=0,1, \ldots$

$$
d_{n}^{(0)}=1, \quad d_{n}^{(k)}=\prod_{1 \leq i \leq j \leq k} \frac{i+j-1+2 n}{i+j-1} \quad \text { for } k, n \geq 1 .
$$


We are going to derive the identities (1.4) and (1.5) simultaneously in the next section.

Our main interest, however, concerns a further generalization of the Catalan numbers and their combinatorial interpretations.

In Section III we shall study Hankel matrices whose entries are defined as generalized Catalan numbers $c_{m}=\frac{1}{3 m+1}\left(\begin{array}{c}3 m+1 \\ m\end{array}\right)$. In this case we could show that

$$
d_{n}^{(0)}=\prod_{j=0}^{n-1} \frac{(3 j+1)(6 j) !(2 j) !}{(4 j+1) !(4 j) !}, \quad d_{n}^{(1)}=\prod_{j=1}^{n} \frac{\left(\begin{array}{c}
6 j-2 \\
2 j
\end{array}\right)}{2\left(\begin{array}{c}
4 j-1 \\
2 j
\end{array}\right)} .
$$

These numbers are of special interest, since they coincide with two Mills - Robbins - Rumsey determinants, which occur in the enumeration of cyclically symmetric plane partitions and alternating sign matrices which are invariant under a reflection about a vertical axis. The relation between Hankel matrices and alternating sign matrices will be discussed in Section IV.

Let us recall some properties of Hankel matrices. Of special importance is the equation

$$
\left(\begin{array}{ccccc}
c_{0} & c_{1} & c_{2} & \ldots & c_{n-1} \\
c_{1} & c_{2} & c_{3} & \ldots & c_{n} \\
c_{2} & c_{3} & c_{4} & \ldots & c_{n+1} \\
\vdots & \vdots & \vdots & & \vdots \\
c_{n-1} & c_{n} & c_{n+1} & \ldots & c_{2 n-2}
\end{array}\right) \cdot\left(\begin{array}{c}
a_{n, 0} \\
a_{n, 1} \\
a_{n, 2} \\
\vdots \\
a_{n, n-1}
\end{array}\right)=\left(\begin{array}{c}
-c_{n} \\
-c_{n+1} \\
-c_{n+2} \\
\vdots \\
-c_{2 n-1}
\end{array}\right)
$$

It is known (cf. [16], p. 246) that, if the matrices $A_{n}^{(0)}$ are nonsingular for all $n$, then the polynomials

$$
t_{j}(x):=x^{j}+a_{j, j-1} x^{j-1}+a_{j, j-2} x^{j-2}+\ldots a_{j, 1} x+a_{j, 0}
$$

form a sequence of monic orthogonal polynomials with respect to the linear operator $T$ mapping $x^{l}$ to its moment $T\left(x^{l}\right)=c_{l}$ for all $l$, i. e.

$$
T\left(t_{j}(x) \cdot t_{m}(x)\right)=0 \text { for } j \neq m \text {. }
$$

and that

$$
T\left(x^{m} \cdot t_{j}(x)\right)=0 \text { for } m=0, \ldots, j-1 .
$$

In Section $\mathrm{V}$ we shall study matrices $L_{n}=(l(m, j))_{m, j=0,1, \ldots, n-1}$ defined by

$$
l(m, j)=T\left(x^{m} \cdot t_{j}(x)\right)
$$

By (1.10) these matrices are lower triangular. The recursion for Catalan - like numbers, as defined by Aigner [2] yielding another generalization of Catalan numbers, can be derived via matrices $L_{n}$ with determinant 1 . Further, the Lanczos algorithm as discussed in [13] yields a factorization $L_{n}=A_{n} \cdot U_{n}^{t}$, where $A_{n}$ is a nonsingular Hankel matrix as in (1.1), $L_{n}$ is defined by (1.11) and 


$$
U_{n}=\left(\begin{array}{cccccc}
1 & 0 & 0 & \ldots & 0 & 0 \\
a_{1,0} & 1 & 0 & \ldots & 0 & 0 \\
a_{2,0} & a_{2,1} & 1 & \ldots & 0 & 0 \\
\vdots & \vdots & \vdots & & \vdots & \vdots \\
a_{n-1,0} & a_{n-1,1} & a_{n-2,2} & \ldots & a_{n-1, n-2} & 1
\end{array}\right)
$$

is the triangular matrix whose entries are the coefficients of the polynomials $t_{j}(x), j=$ $0, \ldots, n-1$.

In Section V we further shall discuss the Berlekamp - Massey algorithm for the decoding of $\mathrm{BCH}$-codes, where Hankel matrices of syndromes resulting after the transmission of a code word over a noisy channel have to be studied. Via the matrix $L_{n}$ defined by (1.11) it will be shown that the Berlekamp - Massey algorithm applied to Hankel matrices with real entries can be used to compute the coefficients in the corresponding orthogonal polynomials and the three - term recurrence defining these polynomials.

Several methods to find Hankel determinants are presented in [61]. We shall mainly concentrate on their occurrence in the theory of continued fractions and orthogonal polynomials. If not mentioned otherwise, we shall always assume that all Hankel matrices $A_{n}$ under consideration are nonsingular.

Hankel matrices come into play when the power series

$$
F(x)=c_{0}+c_{1} x+c_{2} x^{2}+\ldots
$$

is expressed as a continued fraction. If the Hankel determinants $d_{n}^{(0)}$ and $d_{n}^{(1)}$ are different from 0 for all $n$ the so-called $\mathrm{S}$-fraction expansion of $1-x F(x)$ has the form

$$
1-x F(x)=1-\frac{c_{0} x}{1-\frac{q_{1} x}{1-\frac{e_{1} x}{1-\frac{q_{2} x}{1-\frac{e_{2} x}{1-\ldots}}}}} .
$$

Namely, then (cf. [55], p. 304 or [78], p. 200) for $n \geq 1$ and with the convention $d_{0}^{(k)}=1$ for all $k$ it is

$$
q_{n}=\frac{d_{n}^{(1)} \cdot d_{n-1}^{(0)}}{d_{n-1}^{(1)} \cdot d_{n}^{(0)}}, \quad e_{n}=\frac{d_{n+1}^{(0)} \cdot d_{n-1}^{(1)}}{d_{n}^{(0)} \cdot d_{n}^{(1)}}
$$

For the notion of S- and J- fraction (S stands for Stieltjes, J for Jacobi) we refer to the standard books by Perron [55] and Wall [78]. We follow here mainly the $\left(q_{n}, e_{n}\right)$-notation of Rutishauser [65].

For many purposes it is more convenient to consider the variable $\frac{1}{x}$ in (1.13) and study power series of the form 


$$
\frac{1}{x} F\left(\frac{1}{x}\right)=\frac{c_{0}}{x}+\frac{c_{1}}{x^{2}}+\frac{c_{2}}{x^{3}}+\ldots
$$

and its continued S-fraction expansion

$$
\frac{c_{0}}{x-\frac{q_{1}}{1-\frac{e_{1}}{x-\frac{q_{2}}{1-\frac{e_{2}}{x-\ldots}}}}}
$$

which can be transformed to the J-fraction

$$
\frac{c_{0}}{x-\alpha_{1}-\frac{\beta_{1}}{x-\alpha_{2}-\frac{\beta_{2}}{x-\alpha_{3}-\frac{\beta_{3}}{x-\alpha_{4}-\ldots}}}}
$$

with $\alpha_{1}=q_{1}$, and $\alpha_{j+1}=q_{j+1}+e_{j}, \quad \beta_{j}=q_{j} e_{j}$ for $j \geq 1$. (cf. [55], p.375 or [65], pp. $13)$.

The J-fraction corresponding to (1.14) was used by Flajolet ([26] and [27]) to study combinatorial aspects of continued fractions, especially he gave an interpretation of the coefficients in the continued fractions expansion in terms of weighted lattice paths. This interpretation extends to parameters of the corresponding orthogonal polynomials as studied by Viennot [76]. For further combinatorial aspects of orthogonal polynomials see e.g. [28], [72].

Hankel determinants occur in Padé approximation and the determination of the eigenvalues of a matrix using their Schwarz constants, cf. [65]. Especially, they have been studied by Stieltjes in the theory of moments $([70],[71])$. He stated the problem to find out if a measure $\mu$ exists such that

$$
\int_{0}^{\infty} x^{l} d \mu(x)=c_{l} \quad \text { for all } l=0,1, \ldots
$$

for a given sequence $c_{0}, c_{1}, c_{2}, \ldots$ by the approach $\int \frac{d \mu(t)}{x+t}=\sum_{l=0}^{\infty}(-1)^{l} \frac{c_{l}}{x^{l+1}}$.

Stieltjes could show that such a measure exists if the determinants of the Hankel matrices $A_{n}^{(0)}$ and $A_{n}^{(1)}$ are positive for all $n$. Indeed, then (1.9) results from the quality of the approximation to $(1.16)$ by quotients of polynomials $\frac{p_{j}(x)}{t_{j}(x)}$ where $t_{j}(x)$ are just the polynomials (1.8). Hence they obey the three - term recurrence

$$
t_{j}(x)=\left(x-\alpha_{j}\right) t_{j-1}(x)-\beta_{j-1} \cdot t_{j-2}(x), \quad t_{0}(x)=1, \quad t_{1}(x)=x-\alpha_{1},
$$

where

$$
\alpha_{1}=q_{1}, \text { and } \alpha_{j+1}=q_{j+1}+e_{j}, \quad \beta_{j}=q_{j} e_{j} \text { for } j \geq 1 \text {. }
$$


In case that we consider Hankel matrices of the form (1.2) and hence the corresponding power series $c_{k}+c_{k+1} x+c_{k+2} x^{2}+\ldots$, we introduce a superscript (k) to the parameters in question.

Hence, $q_{n}^{(k)}$ and $e_{n}^{(k)}$ denote the coefficients in the continued fractions expansions

$$
\frac{c_{k}}{1-\frac{q_{1}^{(k)} x}{1-\frac{e_{1}^{(k)} x}{1-\frac{q_{2}^{(k)} x}{1-\ldots}}}}, \quad x-q_{1}^{(k)}-\frac{c_{k}}{x-q_{2}^{(k)}-e_{1}^{(k)}-\frac{e_{1}^{(k)} q_{1}^{(k)}}{x-q_{3}^{(k)}-e_{2}^{(k)}-\ldots} q_{2}^{(k)}}
$$

and

$$
t_{j}^{(k)}(x)=x^{j}+a_{j, j-1}^{(k)} x^{j-1}+a_{j, j-2}^{(k)} x^{j-2}+\ldots a_{j, 1}^{(k)} x+a_{j, 0}^{(k)}
$$

are the corresponding polynomials obeying the three - term recurrence

$$
t_{j}^{(k)}(x)=\left(x-\alpha_{j}^{(k)}\right) t_{j-1}^{(k)}(x)-\beta_{j-1}^{(k)} t_{j-2}^{(k)}(x) .
$$

Several algorithms are known to determine this recursion. We mentioned already the Berlekamp - Massey algorithm and the Lanczos algorithm. In the quotient-difference algorithm due to Rutishauser [65] the parameters $q_{n}^{(k)}$ and $e_{n}^{(k)}$ are obtained via the socalled rhombic rule

$$
\begin{gathered}
e_{n}^{(k)}=e_{n-1}^{(k)}+q_{n}^{(k+1)}-q_{n}^{(k)}, \quad e_{0}^{(k)}=0 \text { for all } k, \\
q_{n+1}^{(k)}=q_{n}^{(k+1)} \cdot \frac{e_{n}^{(k+1)}}{e_{n}^{(k)}}, \quad q_{1}^{(k)}=\frac{c_{k+1}}{c_{k}} \text { for all } k .
\end{gathered}
$$

\section{Hankel Matrices and Chebyshev Polynomials}

Let us illustrate the methods introduced by computing determinants of Hankel matrices whose entries are successive Catalan numbers. In several recent papers (e.g. [2], [47], [54], [62]) these determinants have been studied under various aspects and formulae were given for special parameters. Desainte-Catherine and Viennot in [24] provided the general solution $d_{n}^{(k)}=\prod_{1 \leq i \leq j \leq k-1} \frac{i+j+2 n}{i+j}$ for all $n$ and $k$. This was derived as a companion formula (yielding a "90\% bijective proof" for tableaux whose columns consist of an even number of elements and are bounded by height $2 n$ ) to Gordon's result [36] in the proof of the Bender - Knuth conjecture [8]. Gordon proved that $\prod_{1 \leq i \leq j \leq k} \frac{c+i+j-1}{i+j-1}$ is the generating function for Young tableaux with entries from $\{1, \ldots, n\}$ strictly increasing in rows and not decreasing in columns consisting of $\leq c$ columns and largest part $\leq k$. Actually, this follows from the more general formula in the Bender - Knuth conjecture by letting $q \rightarrow 1$, see also [67], p. 265.

By refining the methods of [24], Choi and Gouyou - Beauchamps [21] could also derive Gordon's formula for even $c=2 n$. In the following proposition we shall apply a well - 
known recursion for Hankel determinants allowing to see that in this case also Gordon's formula can be expressed as a Hankel determinant, namely the matrices then consist of consecutive binomial coefficients of the form $\left(\begin{array}{c}2 m+1 \\ m\end{array}\right)$. Simultaneously, this yields another proof of the result of Desainte - Catherine and Viennot, which was originally obtained by application of the quotient - difference algorithm [77].

\section{Proposition 2.1:}

a) For the sequence $c_{m}=\frac{1}{2 m+1}\left(\begin{array}{c}2 m+1 \\ m\end{array}\right), m=0,1, \ldots$ of Catalan numbers it is

$$
d_{n}^{(0)}=d_{n}^{(1)}=1, \quad d_{n}^{(k)}=\prod_{1 \leq i \leq j \leq k-1} \frac{i+j+2 n}{i+j} \quad \text { for } k \geq 2, n \geq 1 .
$$

b) For the binomial coefficients $c_{m}=\left(\begin{array}{c}2 m+1 \\ m\end{array}\right), m=0,1, \ldots$

$$
d_{n}^{(0)}=1, \quad d_{n}^{(k)}=\prod_{1 \leq i \leq j \leq k} \frac{i+j-1+2 n}{i+j-1} \quad \text { for } k, n \geq 1 .
$$

Proof: The proof is based on the following identity for Hankel determinants.

$$
d_{n}^{(k+1)} \cdot d_{n}^{(k-1)}-d_{n-1}^{(k+1)} \cdot d_{n+1}^{(k-1)}-\left[d_{n}^{(k)}\right]^{2}=0 .
$$

This identity can for instance be found in the book by Polya and Szegö [59], Ex. 19, p. 102. It is also an immediate consequence of Dodgson's algorithm for the evaluation of determinants (e.g. [82]).

We shall derive both results simultaneously. The proof will proceed by induction on $n+k$. It is well known, e.g. [69], that for the Hankel matrices $A_{n}^{(k)}$ with Catalan numbers as entries it is $d_{n}^{(0)}=d_{n}^{(1)}=1$. For the induction beginning it must also be verified that $d_{n}^{(2)}=n+1$ and that $d_{n}^{(3)}=\frac{(n+1)(n+2)(2 n+3)}{6}$ is the sum of squares, cf. [47], which can also be easily seen by application of recursion (2.3).

Furthermore, for the matrix $A_{n}^{(k)}$ whose entries are the binomial coefficients $\left(\begin{array}{c}2 k+1 \\ k\end{array}\right),\left(\begin{array}{c}2 k+3 \\ k+1\end{array}\right)$, ... it was shown in [2] that $d_{n}^{(0)}=1$ and $d_{n}^{(1)}=2 n+1$. Application of (2.3) shows that $d_{n}^{(2)}=\frac{(n+1)(2 n+1)(2 n+3)}{3}$, i. e., the sum of squares of the odd positive integers.

Also, it is easily seen by comparing successive quotients $\frac{c_{k+1}}{c_{k}}$ that for $n=1$ the product in (2.1) yields the Catalan numbers and the product in (2.2) yields the binomial coefficients $\left(\begin{array}{c}2 k+1 \\ k+1\end{array}\right)$, cf. also [24].

Now it remains to be verified that (2.1) and (2.2) hold for all $n$ and $k$, which will be done by checking recursion (2.3). The sum in (2.3) is of the form (with either $d=0$ for (2.1) or $d=1$ for $(2.2)$ and shifting $k$ to $k+1$ in $(2.1)$ )

$$
\begin{gathered}
\prod_{i, j=1}^{k} \frac{i+j-d+2 n}{i+j-d} \cdot \prod_{i, j=1}^{k-2} \frac{i+j-d+2 n}{i+j-d}-\prod_{i, j=1}^{k} \frac{i+j-d+2(n+1)}{i+j-d} \cdot \prod_{i, j=1}^{k-2} \frac{i+j-d+2(n-1)}{i+j-d}- \\
-\left[\prod_{i, j=1}^{k-1} \frac{i+j-d+2 n}{i+j-d}\right]^{2}
\end{gathered}
$$




$$
\begin{gathered}
=\left[\prod_{i, j=1}^{k-1} \frac{i+j-d+2 n}{i+j-d}\right]^{2} \cdot \\
\left(\frac{\prod_{j=1}^{k}(k+j-d+2 n) \cdot \prod_{j=1}^{k-1}(k-1+j-d)}{\prod_{j=1}^{k}(k+j-d) \cdot \prod_{j=1}^{k-1}(k-1+j-d+2 n)}-\frac{\prod_{j=0}^{k-1}(j-d+2 n) \cdot \prod_{j=1}^{k-1}(k-1+j-d)}{\prod_{j=1}^{k}(k+j-d) \cdot \prod_{j=1}^{k-1}(1+j-d+2 n)}-1\right) \\
=\left[\prod_{i, j=1}^{k-1} \frac{i+j-d+2 n}{i+j-d}\right]^{2} \cdot \\
\cdot\left(\frac{(2 n+2 k-d)(2 n+2 k-1-d)(k-d)}{(2 n+k-d)(2 k-d)(2 k-1-d)}-\frac{(2 n-d)(2 n+1-d)(k-d)}{(2 n+k-d)(2 k-d)(2 k-1-d)}-1\right) .
\end{gathered}
$$

This expression is 0 exactly if

$$
\begin{gathered}
(2 n+2 k-d)(2 n+2 k-1-d)(k-d)-(2 n-d)(2 n+1-d)(k-d)- \\
-(2 n+k-d)(2 k-d)(2 k-1-d)=0 .
\end{gathered}
$$

In order to show (2.1), now observe that here $d=0$ and then it is easily verified that

$$
(n+k)(2 n+2 k-1)-n(2 n+1)-(2 n+k)(2 k-1)=0 .
$$

In order to show (2.2), we have to set $d=1$ and again the analysis simplifies to verifying

$$
(2 n+2 k-1)(n+k-1)-(2 n-1) n-(2 n+k-1)(2 k-1)=0 .
$$

\section{Remarks:}

1) As pointed out in the introduction, Desainte-Catherine and Viennot [24] derived identity (2.1) and recursion (2.3) simultaneously proves $(2.2)$. The identity $\operatorname{det}\left(A_{n}^{(0)}\right)=1$, when the $c_{m}$ 's are Catalan numbers or binomial coefficients $\left(\begin{array}{c}2 m+1 \\ m\end{array}\right)$ can already be found in [52], pp. $435-436 . d_{n}^{(1)}, d_{n}^{(2)}$, and $d_{n}^{(3)}$ for this case were already mentioned in the proof of Theorem 2.1. The next determinant in this series is obtained via $\frac{d_{n}^{(4)}}{d_{n-1}^{(4)}}=\frac{d_{n+1}^{(3)}}{d_{n-1}^{(3)}}$. For the Catalan numbers then $d_{n}^{(4)}=\frac{d_{n+1}^{(3)} \cdot d_{n}^{(3)}}{5}=\frac{n(n+1)^{2}(n+2)(2 n+1)(2 n+3)}{180}$.

2) Formula (2.1) was also studied by Desainte-Catherine and Viennot [24] in the analysis of disjoint paths in a bounded area of the integer lattice and perfect matchings in a certain graph as a special Pfaffian. An interpretation of the determinant $d_{n}^{(k)}$ in (2.1) as the number of $k$-tuples of disjoint positive lattice paths (see the next section) was used to construct bijections to further combinatorial configurations. Applications of (2.1) in Physics have been discussed by Guttmann, Owczarek, and Viennot [40].

3) The central argument in the proof of Theorem 2.1 was the application of recursion (2.3). Let us demonstrate the use of this recursion with another example. Aigner [3] could show that the Bell numbers are the unique sequence $\left(c_{m}\right)_{m=0,1,2, \ldots}$ such that 


$$
\operatorname{det}\left(A_{n}^{(0)}\right)=\operatorname{det}\left(A_{n}^{(1)}\right)=\prod_{k=0}^{n} k !, \quad \operatorname{det}\left(A_{n}^{(2)}\right)=r_{n+1} \prod_{k=0}^{n} k !,
$$

where $r_{n}=1+\sum_{l=1}^{n} n(n-1) \cdots(n-l+1)$ is the total number of permutations of $n$ things (for $\operatorname{det}\left(A_{n}^{(0)}\right)$ and $\operatorname{det}\left(A_{n}^{(1)}\right)$ see [27] and [23]). In [3] an approach via generating functions was used in order to derive $d_{n}^{(2)}=\operatorname{det}\left(A_{n}^{(2)}\right)$ in (2.4). Setting $d_{n}^{(2)}=r_{n+1} \cdot \prod_{k=0}^{n} k$ ! in (2.4), with (2.3) one obtains the recurrence $r_{n+1}=(n+1) \cdot r_{n}+1, r_{2}=5$, which just characterizes the total number of permutations of $n$ things, cf. [63], p. 16, and hence can $\operatorname{derive} \operatorname{det}\left(A_{n}^{(2)}\right)$ from $\operatorname{det}\left(A_{n}^{(0)}\right)$ and $\operatorname{det}\left(A_{n}^{(1)}\right)$ also this way.

4) From the proof of Proposition 2.1 it is also clear that $\prod_{1 \leq i, j \leq k} \frac{i+j-d+2 n}{i+j-d}$ yields a sequence of Hankel determinants $d_{n}^{(k)}$ only for $d=0,1$, since otherwise recursion (2.3) is not fulfilled.

As pointed out, in [24] formula (2.1) was derived by application of the quotient - difference algorithm, cf. also [21] for a more general result. The parameters $q_{n}^{(k)}$ and $e_{n}^{(k)}$ also can be obtained from Proposition 2.1.

Corollary 2.1: For the Catalan numbers the coefficients $q_{n}^{(k)}$ and $e_{n}^{(k)}$ in the continued fractions expansion of $\sum_{m=0}^{\infty} \frac{1}{2(k+m)+1}\left(\begin{array}{c}2(k+m)+1 \\ k+m\end{array}\right) x^{m}$ as in (1.14) are given as

$$
q_{n}^{(k)}=\frac{(2 n+2 k-1)(2 n+2 k)}{(2 n+k-1)(2 n+k)}, \quad e_{n}^{(k)}=\frac{(2 n)(2 n+1)}{(2 n+k)(2 n+k+1)} .
$$

For the binomial coefficients $\left(\begin{array}{c}2 m+1 \\ m\end{array}\right)$ the corresponding coefficients in the expansion of $\sum_{m=0}^{\infty}\left(\begin{array}{c}2(k+m)+1 \\ k+m\end{array}\right) x^{m}$ are

$$
q_{n}^{(k)}=\frac{(2 n+2 k)(2 n+2 k+1)}{(2 n+k-1)(2 n+k)}, \quad e_{n}^{(k)}=\frac{(2 n-1)(2 n)}{(2 n+k)(2 n+k+1)} .
$$

Proof: (2.5) and (2.6) can be derived by application of the rhombic rule (1.21) and (1.22). They are also immediate from the previous Proposition 2.1 by application of (1.15), which for $k>0$ generalizes to the following formulae from [65], p. 15, where the $d_{n}^{(k)}$, s are Hankel determinants as (1.3).

$$
q_{n}^{(k)}=\frac{d_{n}^{(k+1)} d_{n-1}^{(k)}}{d_{n}^{(k)} d_{n-1}^{(k+1)}}, \quad e_{n}^{(k)}=\frac{d_{n+1}^{(k)} d_{n-1}^{(k)}}{d_{n}^{(k)} d_{n}^{(k+1)}}
$$

Corollary 2.2: The orthogonal polynomials associated to the Hankel matrices $A_{n}^{(k)}$ of Catalan numbers $c_{m}=\frac{1}{2 m+1}\left(\begin{array}{c}2 m+1 \\ m\end{array}\right)$ are

$$
t_{n}^{(k)}(x)=\left(x-\alpha_{n}^{(k)}\right) t_{n-1}^{(k)}-\beta_{n-1}^{(k)} t_{n-2}^{(k)}(x), \quad t_{0}^{(k)}(x)=1, \quad t_{1}^{(k)}(x)=x-\frac{4 k+2}{k+2}
$$

where 


$$
\alpha_{n+1}^{(k)}=2-\frac{2 k(k-1)}{(2 n+k+2)(2 n+k)}, \quad \beta_{n}^{(k)}=\frac{(2 n+2 k-1)(2 n+2 k)(2 n)(2 n+1)}{(2 n+k-1)(2 n+k)^{2}(2 n+k+1)}
$$

Proof: By $(1.20), \beta_{n}^{(k)}=q_{n}^{(k)} \cdot e_{n}^{(k)}$ as in the previous corollary and

$$
\begin{gathered}
\alpha_{n+1}^{(k)}=q_{n+1}^{(k)}+ \\
+e_{n}^{(k)}=\frac{(2 n+2 k+1)(2 n+2 k+2)((2 n+k)+(2 n)(2 n+1)(2 n+k+2)}{(2 n+k+1)(2 n+k+2)(2 n+k)} \\
=\frac{8 n^{2}+8 n k+8 n+2 k+4 k^{2}}{(2 n+k+2)(2 n+k)}=2-\frac{2 k(k-1)}{(2 n+k+2)(2 n+k)} .
\end{gathered}
$$

Especially for small parameters $k$ the following families of orthogonal polynomials arise here.

$$
\begin{gathered}
t_{n}^{(0)}(x)=(x-2) \cdot t_{n-1}^{(0)}(x)-t_{n-2}^{(0)}(x), \quad t_{0}^{(0)}(x)=1, \quad t_{1}^{(0)}(x)=x-1, \\
t_{n}^{(1)}(x)=(x-2) \cdot t_{n-1}^{(1)}(x)-t_{n-2}^{(1)}(x), \quad t_{0}^{(1)}(x)=1, \quad t_{1}^{(1)}(x)=x-2, \\
t_{n}^{(2)}(x)=\left(x-\frac{(n+1)^{2}+n^{2}}{n(n+1)}\right) \cdot t_{n-1}^{(2)}(x)-\frac{n^{2}-1}{n^{2}} t_{n-2}^{(2)}(x), \quad t_{0}^{(2)}(x)=1, t_{1}^{(2)}(x)=x-\frac{5}{2} .
\end{gathered}
$$

It is well - known that the Chebyshev - polynomials of the second kind

$$
u_{n}(x)=\sum_{i=0}^{\left\lfloor\frac{n}{2}\right\rfloor}(-1)^{i}\left(\begin{array}{c}
n-i \\
i
\end{array}\right)(2 x)^{n-2 i}
$$

with recursion

$$
u_{n}(x)=2 x \cdot u_{n-1}(x)-u_{n-2}(x), \quad u_{0}(x)=1, \quad u_{1}(x)=2 x
$$

come in for Hankel matrices with Catalan numbers as entries. For instance, in this case the first orthogonal polynomials in Corollary 2.2 are

$$
t_{n}^{(0)}\left(x^{2}\right)=\frac{1}{x} u_{2 n}\left(\frac{x}{2}\right), \quad t_{n}^{(1)}\left(x^{2}\right)=\frac{1}{x} u_{2 n+1}\left(\frac{x}{2}\right) .
$$

Corollary 2.3: The orthogonal polynomials associated to the Hankel matrices $A_{n}^{(k)}$ of binomial coefficients $c_{m}=\left(\begin{array}{c}2 m+1 \\ m\end{array}\right)$ are

$$
t_{n}^{(k)}(x)=\left(x-\alpha_{n}^{(k)}\right) t_{n-1}^{(k)}-\beta_{n-1}^{(k)} t_{n-2}^{(k)}(x), \quad t_{0}^{(k)}(x)=1, \quad t_{1}^{(k)}(x)=x-\frac{4 k+6}{k+2}
$$

where

$$
\alpha_{n+1}^{(k)}=2-\frac{2 k(k+1)}{(2 n+k+2)(2 n+k)}, \quad \beta_{n+1}^{(k)}=\frac{(2 n+2 k)(2 n+2 k+1)(2 n-1)(2 n)}{(2 n+k-1)(2 n+k)^{2}(2 n+k+1)} .
$$


Proof: Again, $\beta_{n}^{(k)}=q_{n}^{(k)} \cdot e_{n}^{(k)}$ as in the previous corollary and

$$
\begin{gathered}
\alpha_{n+1}^{(k)}=q_{n+1}^{(k)}+ \\
+e_{n}^{(k)}=\frac{(2 n+2 k+2)(2 n+2 k+3)((2 n+k)+(2 n-1)(2 n)(2 n+k+2)}{(2 n+k)(2 n+k+1)(2 n+k+2)} \\
=\frac{8 n^{2}+8 n k+8 n+2 k^{2}+4 k}{(2 n+k+2)(2 n+k)}=2-\frac{2 k(k+1)}{(2 n+k+2)(2 n+k)} .
\end{gathered}
$$

\section{Generalized Catalan Numbers And Hankel Determinants}

For an integer $p \geq 2$ we shall denote the numbers $\frac{1}{p m+1}\left(\begin{array}{c}p m+1 \\ m\end{array}\right)$ as generalized Catalan numbers. The Catalan numbers occur for $p=2$. (The notion "generalized Catalan numbers" as in [42] is not standard, for instance, in [39], pp. $344-350$ it is suggested to denote them "Fuss numbers").

Their generating function

$$
C_{p}(x)=\sum_{m=0}^{\infty} \frac{1}{p m+1}\left(\begin{array}{c}
p m+1 \\
m
\end{array}\right) x^{m}
$$

fulfills the functional equation

$$
C_{p}(x)=1+x \cdot C_{p}(x)^{p}
$$

from which immediately follows that

$$
\frac{1}{C_{p}(x)}=1-x \cdot C_{p}(x)^{p-1}
$$

Further, it is

$$
C_{p}(x)^{p-1}=\sum_{m=0}^{\infty} \frac{1}{p m+p-1}\left(\begin{array}{c}
p m+p-1 \\
m+1
\end{array}\right) x^{m} .
$$

It is well known that the generalized Catalan numbers $\frac{1}{p m+1}\left(\begin{array}{c}p m+1 \\ m\end{array}\right)$ count the number of paths in the integer lattice $\mathbb{Z} \times \mathbb{Z}$ (with directed vertices from $(i, j)$ to either $(i, j+1)$ or to $(i+1, j))$ from the origin $(0,0)$ to $(m,(p-1) m)$ which never go above the diagonal $(p-1) x=y$. Equivalently, they count the number of paths in $\mathbb{Z} \times \mathbb{Z}$ starting in the origin $(0,0)$ and then first touching the boundary $\{(l+1,(p-1) l+1): l=0,1,2, \ldots\}$ in $(m,(p-1) m+1)$ (cf. e.g. [75]).

Viennot [76] gave a combinatorial interpretation of Hankel determinants in terms of disjoint Dyck paths. In case that the entries of the Hankel matrix are consecutive Catalan numbers this just yields an equivalent enumeration problem analyzed by Mays and Wojciechowski [47]. The method of proof from [47] extends to Hankel matrices consisting of generalized Catalan numbers as will be seen in the following proposition.

Proposition 3.1: If the $c_{m}$ 's in (1.2) are generalized Catalan numbers, $c_{m}=\frac{1}{p m+1}\left(\begin{array}{c}p m+1 \\ m\end{array}\right)$, $p \geq 2$ a positive integer, then $\operatorname{det}\left(A_{n}^{(k)}\right)$ is the number of $n$-tuples $\left(\gamma_{0}, \ldots, \gamma_{n-1}\right)$ of vertex 
- disjoint paths in the integer lattice $\mathbb{Z} \times \mathbb{Z}$ (with directed vertices from $(i, j$ ) to either $(i, j+1)$ or to $(i+1, j))$ never crossing the diagonal $(p-1) x=y$, where the path $\gamma_{r}$ is from $(-r,-(p-1) r)$ to $(k+r,(p-1)(k+r))$.

Proof: The proof follows the same lines as the one in [32], which was carried out only for the case $p=2$ and is based on a result in [46] on disjoint path systems in directed graphs. We follow here the presentation in [47].

Namely, let $\mathcal{G}$ be an acyclic directed graph and let $\mathcal{A}=\left\{a_{0}, \ldots, a_{n-1}\right\}, \mathcal{B}=\left\{b_{0}, \ldots, b_{n-1}\right\}$ be two sets of vertices in $\mathcal{G}$ of the same size $n$. A disjoint path system in $(\mathcal{G}, \mathcal{A}, \mathcal{B})$ is a system of vertex disjoint paths $\left(\gamma_{0}, \ldots, \gamma_{n-1}\right)$, where for every $i=0, \ldots, n-1$ the path $\gamma_{i}$ leads from $a_{i}$ to $b_{\sigma(i)}$ for some permutation $\sigma$ on $\{0, \ldots, n-1\}$.

Now let $p_{i j}$ denote the number of paths leading from $a_{i}$ to $b_{j}$ in $\mathcal{G}$, let $p^{+}$be the number of disjoint path systems for which $\sigma$ is an even permutation and let $p^{-}$be the number of disjoint path systems for which $\sigma$ is an odd permutation. Then $\operatorname{det}\left(\left(p_{i j}\right)_{i, j=0, \ldots, n-1}\right)=$ $p^{+}-p^{-}$(Theorem 3 in [47]).

Now consider the special graph $\mathcal{G}^{\prime}$ with vertex set

$$
\mathcal{V}=\{(u, v) \in \mathbb{Z} \times \mathbb{Z}:(p-1) u \leq v\},
$$

i. e. the part of the integer lattice on and above the diagonal $(p-1) x=y$, and directed edges connecting $(u, v)$ to $(u, v+1)$ and to $(u+1, v)$ (if this is in $\mathcal{V}$, of course).

Further let $\mathcal{A}=\left\{a_{0}, \ldots a_{n-1}\right\}$ and $\mathcal{B}=\left\{b_{0}, \ldots b_{n-1}\right\}$ be two sets disjoint to each other and to $\mathcal{V}$. Then we connect $\mathcal{A}$ and $\mathcal{B}$ to $\mathcal{G}^{\prime}$ by introducing directed edges as follows

$$
a_{i} \rightarrow(-i,-(p-1) i), \quad(k+i,(p-1)(k+i)) \rightarrow b_{i}, \quad i=0, \ldots, n-1 .
$$

Now denote by $\mathcal{G}^{\prime \prime}$ the graph with vertex set $\mathcal{V} \cup \mathcal{A} \cup \mathcal{B}$ whose edges are those from $\mathcal{G}^{\prime}$ and the additional edges connecting $\mathcal{A}$ and $\mathcal{B}$ to $\mathcal{G}^{\prime}$ as described in (3.4).

Observe that any permutation $\sigma$ on $\{0, \ldots, n-1\}$ besides the identity would yield some $j$ and $l$ with $\sigma(j)>j$ and $\sigma(l)<l$. But then the two paths $\gamma_{j}$ from $a_{j}$ to $b_{\sigma(j)}$ and $\gamma_{l}$ from $a_{l}$ to $b_{\sigma(l)}$ must cross and hence share a vertex. So the only permutation yielding a disjoint path system for $\mathcal{G}^{\prime}$ is the identity. The number of paths $p_{i j}$ from $a_{i}$ to $b_{j}$ is the generalized Catalan number $\frac{1}{p(k+i+j)+1}\left(\begin{array}{c}p(k+i+j)+1 \\ (k+i+j)\end{array}\right)$. So the matrix $\left(p_{i j}\right)$ is of Hankel type as required and its determinant gives the number of $n$-tuples of disjoint paths as described in Proposition 3.1.

\section{Remarks:}

1) The use of determinants in the enumeration of disjoint path systems is well known, e.g. [31]. In a similar way as in Proposition 3.1 we can derive an analogous result for the number of tuples of vertex - disjoint lattice paths, with the difference that the paths now are not allowed to touch the diagonal $(p-1) x=y$ before they terminate in $(m,(p-1) m)$. Since the number of such paths from $(0,0)$ to $(m,(p-1) m)$ is $\frac{1}{p m+p-1}\left(\begin{array}{c}p m+p-1 \\ m+1\end{array}\right)$ (cf. e.g. the appendix), this yields a combinatorial interpretation of Hankel matrices $A_{n}^{(k)}$ with these numbers as entries as in (1.2).

2) For the Catalan numbers, i. e. $p=2$, lattice paths are studied which never cross the diagonal $x=y$. Viennot provided a combinatorial interpretation of orthogonal polynomials 
by assigning weights to the steps in such a path, which are obtained from the coefficients in the three-term recurrence of the orthogonal polynomials ([76], cf also. [26]). In the case that all coefficients $\alpha_{j}$ are 0 , a Dyck path arises with vertical steps having all weight 1 and horizontal steps having weight $\beta_{j}$ for some $j$. For the Catalan numbers as entries in the Hankel matrix all $\beta_{j}$ 's are 1 , since the Chebyshev polynomials of second kind arise. So the total number of all such paths is counted. Observe that Proposition 3.1 extends the path model for the Catalan numbers in another direction, namely the weights of the single steps are still all 1, but the paths now are not allowed to cross a different boundary.

In order to evaluate the Hankel determinants we further need the following identity.

Lemma 3.1: Let $p \geq 2$ be an integer. Then

$$
\left(\sum_{m=0}^{\infty}\left(\begin{array}{c}
p m \\
m
\end{array}\right) x^{m}\right) \cdot\left(\sum_{m=0}^{\infty} \frac{1}{p m+1}\left(\begin{array}{c}
p m+1 \\
m
\end{array}\right) x^{m}\right)=\sum_{m=0}^{\infty}\left(\begin{array}{c}
p m+1 \\
m
\end{array}\right) x^{m} .
$$

Proof: We are obviously done if we could show that for all $m=0,1,2, \ldots$.

$$
\left(\begin{array}{c}
p m+1 \\
m
\end{array}\right)=\sum_{l=0}^{m} \frac{1}{p l+1}\left(\begin{array}{c}
p l+1 \\
l
\end{array}\right) \cdot\left(\begin{array}{c}
p(m-l) \\
m-l
\end{array}\right)
$$

In order to do so, we count the number $\left(\begin{array}{c}p m+1 \\ m\end{array}\right)$ of lattice paths (where possible steps are from $(i, j)$ to either $(i, j+1)$ or to $(i+1, j))$ from $(0,0)$ to $(m,(p-1) m+1)$ in a second way. Namely each such path must go through at least one of the points $(l,(p-1) l+1)$, $l=0,1, \ldots, m$. Now we divide the path into two subpaths, the first subpath leading from the origin $(0,0)$ to the first point of the form $(l,(p-1) l+1)$ and the second subpath from $(l,(p-1) l+1)$ to $(m,(p-1) m+1)$. Recall that there are $\frac{1}{p l+1}\left(\begin{array}{c}p l+1 \\ l\end{array}\right)$ possible choices for the first subpath and obviously there exist $\left(\begin{array}{c}p(m-l) \\ m-l\end{array}\right)$ possibilities for the choice of the second subpath.

Theorem 3.1: For $m=0,1,2 \ldots$ let denote $c_{m}=\frac{1}{3 m+1}\left(\begin{array}{c}3 m+1 \\ m\end{array}\right)$ and $b_{m}=\frac{1}{3 m+2}\left(\begin{array}{c}3 m+2 \\ m+1\end{array}\right)$. Then

$$
\begin{gathered}
\left(\begin{array}{ccccc}
c_{0} & c_{1} & c_{2} & \ldots & c_{n-1} \\
c_{1} & c_{2} & c_{3} & \ldots & c_{n} \\
c_{2} & c_{3} & c_{4} & \ldots & c_{n+1} \\
\vdots & \vdots & \vdots & & \vdots \\
c_{n-1} & c_{n} & c_{n+1} & \ldots & c_{2 n-2}
\end{array}\right)=\prod_{j=0}^{n-1} \frac{(3 j+1)(6 j) !(2 j) !}{(4 j+1) !(4 j) !} \\
\left(\begin{array}{cccccc}
c_{1} & c_{2} & c_{3} & \ldots & c_{n} \\
c_{2} & c_{3} & c_{4} & \ldots & c_{n+1} \\
c_{3} & c_{4} & c_{5} & \ldots & c_{n+2} \\
\vdots & \vdots & \vdots & & \vdots \\
c_{n} & c_{n+1} & c_{n+2} & \ldots & c_{2 n-1}
\end{array}\right)=\prod_{j=1}^{n} \frac{\left(\begin{array}{c}
6 j-2 \\
2 j
\end{array}\right)}{2\left(\begin{array}{c}
4 j-1 \\
2 j
\end{array}\right)}
\end{gathered}
$$


and

$$
\begin{gathered}
\left(\begin{array}{ccccc}
b_{0} & b_{1} & b_{2} & \ldots & b_{n-1} \\
b_{1} & b_{2} & b_{3} & \ldots & b_{n} \\
b_{2} & b_{3} & b_{4} & \ldots & b_{n+1} \\
\vdots & \vdots & \vdots & & \vdots \\
b_{n-1} & b_{n} & b_{n+1} & \ldots & b_{2 n-2}
\end{array}\right)=\prod_{j=1}^{n} \frac{\left(\begin{array}{c}
6 j-2 \\
2 j
\end{array}\right)}{2\left(\begin{array}{c}
4 j-1 \\
2 j
\end{array}\right)}, \\
\left(\begin{array}{ccccc}
b_{1} & b_{2} & b_{3} & \ldots & b_{n} \\
b_{2} & b_{3} & b_{4} & \ldots & b_{n+1} \\
b_{3} & b_{4} & b_{5} & \ldots & b_{n+2} \\
\vdots & \vdots & \vdots & & \vdots \\
b_{n} & b_{n+1} & b_{n+2} & \ldots & b_{2 n-1}
\end{array}\right)=\prod_{j=0}^{n} \frac{(3 j+1)(6 j) !(2 j) !}{(4 j+1) !(4 j) !}
\end{gathered}
$$

Proof: Observe that

$$
\left(\begin{array}{c}
3 m \\
m
\end{array}\right)=\frac{\prod_{j=1}^{m}(3 j) \prod_{j=0}^{m-1}(3 j+1) \prod_{j=0}^{m-1}(3 j+2)}{m ! \prod_{j=1}^{m}(2 j) \prod_{j=0}^{m-1}(2 j+1)}=\left(\frac{27}{4}\right)^{m} \frac{\prod_{j=0}^{m-1}\left(\frac{2}{3}+j\right) \prod_{j=0}^{m-1}\left(\frac{1}{3}+j\right)}{m ! \prod_{j=0}^{m-1}\left(\frac{1}{2}+j\right)}
$$

and accordingly

$$
\left(\begin{array}{c}
3 m+1 \\
m
\end{array}\right)=\frac{\prod_{j=1}^{m}(3 j) \prod_{j=0}^{m-1}(3 j+4) \prod_{j=0}^{m-1}(3 j+2)}{m ! \prod_{j=1}^{m}(2 j) \prod_{j=0}^{m-1}(2 j+3)}=\left(\frac{27}{4}\right)^{m} \frac{\prod_{j=0}^{m-1}\left(\frac{2}{3}+j\right) \prod_{j=0}^{m-1}\left(\frac{4}{3}+j\right)}{m ! \prod_{j=0}^{m-1}\left(\frac{3}{2}+j\right)} .
$$

Then with (3.2) and (3.5) we have the representation

$$
D(x):=1-x \cdot C_{3}(x)^{2}=\frac{\sum_{m=0}^{\infty}\left(\begin{array}{c}
3 m \\
m
\end{array}\right) x^{m}}{\sum_{m=0}^{\infty}\left(\begin{array}{c}
3 m+1 \\
m
\end{array}\right) x^{m}}=\frac{F(\alpha, \beta, \gamma, y)}{F(\alpha, \beta+1, \gamma+1, y)},
$$

which is the quotient of two hypergeometric series, where

$F(\alpha, \beta, \gamma, y)=1+\frac{\alpha \beta}{\gamma} y+\frac{\alpha(\alpha+1) \beta(\beta+1)}{2 ! \cdot \gamma(\gamma+1)} y^{2}+\frac{\alpha(\alpha+1)(\alpha+2) \beta(\beta+1)(\beta+2)}{3 ! \cdot \gamma(\gamma+1)(\gamma+2)} y^{2}+\ldots$

with the parameter choice

$$
\alpha=\frac{2}{3}, \quad \beta=\frac{1}{3}, \quad \gamma=\frac{1}{2}, \quad y=\frac{27}{4} x .
$$

For quotients of such hypergeometric series the continued fractions expansion as in (1.14) was found by Gauss (see [55], p. 311 or [78], p. 337). Namely for $n=1,2, \ldots$ it is

$$
e_{n}=\frac{(\alpha+n)(\gamma-\beta+n)}{(\gamma+2 n)(\gamma+2 n+1)}, \quad q_{n}=\frac{(\beta+n)(\gamma-\alpha+n)}{(\gamma+2 n-1)(\gamma+2 n)}
$$

Now denoting by $q_{n}^{(D)}$ and $e_{n}^{(D)}$ the coefficients in the continued fractions expansion of the power series $D(x)=1-x C_{3}(x)^{2}$ under consideration, then taking into account that $y=\frac{27}{4} x$ we obtain with the parameters in (3.8) that 


$$
e_{n}^{(D)}=\frac{3}{2} \frac{(6 n+1)(3 n+2)}{(4 n+1)(4 n+3)}, \quad q_{n}^{(D)}=\frac{3}{2} \frac{(6 n-1)(3 n+1)}{(4 n-1)(4 n+1)} .
$$

The continued fractions expansion of $1+x C_{3}(x)^{2}$ differs from that of $1-x C_{3}(x)^{2}$ only by changing the sign of $c_{0}$ in (1.14).

So, by application of (1.15) the identity (3.7) for the determinants $d_{n}^{(0)}$ and $d_{n}^{(1)}$ of Hankel matrices with the numbers $\frac{1}{3 m+2}\left(\begin{array}{c}3 m+2 \\ m+1\end{array}\right)$ as entries is easily verified by induction. Namely, observe that

$$
\begin{gathered}
\frac{3}{2} \frac{(6 n-1)(3 n+1)}{(4 n-1)(4 n+1)}=\frac{2(6 n)(6 n-1)(2 n)(3 n+1)}{(4 n+1)(4 n)^{2}(4 n-1)} \\
\quad=\frac{(3 n+1)(6 n) !(2 n) !}{(4 n+1) !(4 n) !} \cdot \frac{2\left(\begin{array}{c}
4 n-1 \\
2 n
\end{array}\right)}{\left(\begin{array}{c}
6 n-2 \\
2 n
\end{array}\right)}=\frac{d_{n}^{(1)}}{d_{n-1}^{(1)}} \cdot \frac{d_{n-1}^{(0)}}{d_{n}^{(0)}}
\end{gathered}
$$

and that

$$
\begin{gathered}
\frac{3}{2} \frac{(6 n+1)(3 n+2)}{(4 n+1)(4 n+3)}=\frac{(6 n+4)(6 n+3)(6 n+2)(6 n+1)(2 n+1)}{2(4 n+3)(4 n+2)^{2}(4 n+1)(3 n+1)} \\
=\frac{\left(\begin{array}{c}
6 n+4 \\
2 n+2
\end{array}\right)}{2\left(\begin{array}{c}
4 n+3 \\
2 n+1
\end{array}\right)} \cdot \frac{(4 n+1) !(4 n) !}{(3 n+1)(6 n) !(2 n) !}=\frac{d_{n+1}^{(0)}}{d_{n}^{(0)}} \cdot \frac{d_{n-1}^{(1)}}{d_{n}^{(1)}}
\end{gathered}
$$

where $d_{n-1}^{(0)}, d_{n-1}^{(1)}, d_{n}^{(0)}, d_{n}^{(1)}, d_{n+1}^{(0)}$ are the determinants for the Hankel matrices in (3.7). In order to find the determinants for the Hankel matrices in (3.6) with generalized Catalan numbers $\frac{1}{3 m+1}\left(\begin{array}{c}3 m+1 \\ m\end{array}\right)$ as entries, just recall that $D(x)=1-x C_{3}(x)^{2}=\frac{1}{C_{3}(x)}$. So the continued fractions expansion of

$$
1+x C_{3}(x)=1-\frac{-x}{1-x C_{3}(x)^{2}}=1-\frac{-x}{1-\frac{q_{1}^{(C)} x}{1-\frac{e_{1}^{(C)} x}{1-\frac{q_{2}^{(C)} x}{1-\ldots}}}}
$$

is obtained by setting $q_{1}^{(C)}=1, e_{n}^{(C)}=q_{n}^{(D)}$ for $n \geq 1$ and $q_{n}^{(C)}=e_{n-1}^{(D)}$ for $n \geq 2$.

Problem: In the last section we were able to derive all Hankel determinants $d_{n}^{(k)}$ with Catalan numbers as entries. So the case $p=2$ for Hankel determinants (1.2) consisting of numbers $\frac{1}{p m+1}\left(\begin{array}{c}p m+1 \\ m\end{array}\right)$ is completely settled. For $p=3$, the above theorem yields $d_{n}^{(0)}$ and $d_{n}^{(1)}$. However the methods do not work in order to determine $d_{n}^{(k)}$ for $k \geq 2$. Also they do not allow to find determinants of Hankel matrices consisting of generalized Catalan numbers when $p \geq 4$. What can be said about these cases?

Let us finally discuss the connection to the Mills - Robbins - Rumsey determinants 


$$
T_{n}(x, \mu)=\operatorname{det}\left(\sum_{t=0}^{2 n-2}\left(\begin{array}{c}
i+\mu \\
t-i
\end{array}\right)\left(\begin{array}{c}
j \\
2 j-t
\end{array}\right) x^{2 j-t}\right)_{i, j=0, \ldots, n-1},
$$

where $\mu$ is a nonnegative integer (discussed e.g. in [50], [6], [5], [22], and [57]). For $\mu=0,1$ it is $T_{n}(1, \mu)=d_{n}^{(\mu)}$ - the Hankel determinants in (3.6). This coincidence does not continue for $\mu \geq 2$.

Using former results by Andrews [4], Mills, Robbins, and Rumsey [50] could derive that

$$
T_{n}(1, \mu)=\operatorname{det}\left(\left(\begin{array}{c}
\mu+i+j \\
2 j-i
\end{array}\right)\right)_{i, j=0, \ldots, n-1}=\frac{1}{2^{n}} \prod_{k=0}^{n-1} \Delta_{2 k}(2 \mu)
$$

where $\Delta_{0}(\mu)=2$ and with $(x)_{j}=x(x+1)(x+2) \cdots(x+j-1)$

$$
\Delta_{2 k}(\mu)=\frac{(\mu+2 k+2)_{k}\left(\frac{1}{2} \mu+2 k+\frac{3}{2}\right)_{k-1}}{(k)_{k}\left(\frac{1}{2} \mu+k+\frac{3}{2}\right)_{k-1}}, \quad k>0 .
$$

They also state that the proof of formula (3.11) is quite complicated and that it would be interesting to find a simpler one. One might look for an approach via continued fractions for further parameters $\mu$, however, application of Gauss's theorem only works for $\mu=0,1$, where (3.9) also follows from (3.11).

Mills, Robbins, and Rumsey [50] found the number of cyclically symmetric plane partitions of size $n$, which are equal to its transpose-complement to be the determinant $T_{n}(1,0)$. They also conjectured $T_{n}(x, 1)$ to be the generating function for alternating sign matrices invariant under a reflection about a vertical axis, especially $T_{n}(1,1)$ should then be the total number of such alternating sign matrices as stated by Stanley [68]. We shall further discuss this conjecture in Section IV.

The determinant $T_{n}(1, \mu)=\operatorname{det}\left(\sum_{t=0}^{2 n-2}\left(\begin{array}{c}i+\mu \\ t-i\end{array}\right)\left(\begin{array}{c}j \\ t-j\end{array}\right)\right)_{i, j=0, \ldots, n-1}$, comes in as counting function for another class of vertex-disjoint path families in the integer lattice. Namely, for such a such a tuple $\left(\gamma_{0}, \ldots, \gamma_{n-1}\right)$ of disjoint paths, path $\gamma_{i}$ leads from $(i, 2 i+\mu)$ to $(2 i, i)$. By a bijection to such disjoint path families for $\mu=0$ the enumeration problem for the above - mentioned family of plane partitions was finally settled in [50].

\section{Alternating Sign Matrices}

An alternating sign matrix is a square matrix with entries from $\{0,1,-1\}$ such that i) the entries in each row and column sum up to 1, ii) the nonzero entries in each row and column alternate in sign. An example is

$$
\left(\begin{array}{rrrrrrr}
0 & 0 & 0 & 1 & 0 & 0 & 0 \\
1 & 0 & 0 & -1 & 0 & 0 & 1 \\
0 & 0 & 0 & 1 & 0 & 0 & 0 \\
0 & 1 & 0 & -1 & 0 & 1 & 0 \\
0 & 0 & 0 & 1 & 0 & 0 & 0 \\
0 & 0 & 1 & -1 & 1 & 0 & 0 \\
0 & 0 & 0 & 1 & 0 & 0 & 0
\end{array}\right)
$$


Robbins and Rumsey discovered the alternating sign matrices in the analysis of Dodgson's algorithm in order to evaluate the determinant of an $n \times n$-matrix. Reverend Charles Lutwidge Dodgson, who worked as a mathematician at the Christ College at the University of Oxford is much wider known as Lewis Carroll, the author of [18]. His algorithm, which is presented in [16], pp. $113-115$, is based on the following identity for any matrix ([25], for a combinatorial proof see [82]).

$$
\begin{gathered}
\operatorname{det}\left(\left(a_{i, j}\right)_{i, j=1, \ldots, n}\right) \cdot \operatorname{det}\left(\left(a_{i, j}\right)_{i, j=2, \ldots, n-1}\right)=\operatorname{det}\left(\left(a_{i, j}\right)_{i, j=1, \ldots, n-1}\right) \cdot \operatorname{det}\left(\left(a_{i, j}\right)_{i, j=2, \ldots, n}\right)- \\
-\operatorname{det}\left(\left(a_{i, j}\right)_{i=1, \ldots, n-1, j=2, \ldots, n}\right) \cdot \operatorname{det}\left(\left(a_{i, j}\right)_{i=2, \ldots, n, j=1, \ldots, n-1}\right) .
\end{gathered}
$$

If $\left(a_{i, j}\right)_{i, j=1, \ldots, n}$ in (4.2) is a Hankel matrix, then all the other matrices in (4.2) are Hankel matrices, too. Hence recursion (2.3) from the introduction is an immediate consequence of Dodgson's result.

In the course of Dodgson's algorithm only $2 \times 2$ determinants have to be calculated. Robbins asked what would happen, if in the algorithm we would replace the determinant evaluation $a_{i j} a_{i+1, j+1}-a_{i, j+1} a_{i+1, j}$ by the prescription $a_{i j} a_{i+1, j+1}+x a_{i, j+1} a_{i+1, j}$, where $x$ is some variable.

It turned out that this yields a sum of monomials in the $a_{i j}$ and their inverses, each monomial multiplied by a polynomial in $x$. The monomials are of the form $\prod_{i, j=1}^{n} a_{i j}^{b_{i j}}$ where the $b_{i j}$ 's are the entries in an alternating sign matrix. The exact formula can be found in Theorem 3.13 in the book "Proofs and Confirmations: The Story of The Alternating Sign Matrix Conjecture" by David Bressoud [16].

The alternating sign matrix conjecture concerns the total number of $n \times n$ alternating sign matrices, which was conjectured by Mills, Robbins, and Rumsey to be $\prod_{j=0}^{n-1} \frac{(3 j+1) !}{(n+j) !}$. The problem was open for fifteen years until it was finally settled by Zeilberger [80]. The development of ideas is described in the book by Bressoud. There are deep relations to various parts of Algebraic Combinatorics, especially to plane partitions, where the same counting function occurred, and also to Statistical Mechanics, where the configuration of water molecules in "square ice" can be described by an alternating sign matrix.

As an important step in the derivation of the refined alternating sign matrix conjecture [81], a Hankel matrix comes in, whose entries are $c_{m}=\frac{1-q^{m+1}}{1-q^{3(m+1)}}$. The relevant orthogonal polynomials in this case are a discrete version of the Legendre polynomials.

Many problems concerning the enumeration of special types of alternating sign matrices are still unsolved, cf. [16], pp. 201. Some of these problems have been presented by Stanley in [68], where it is also conjectured that the number $V(2 n+1)$ of alternating sign matrices of odd order $2 n+1$ invariant under a reflection about a vertical axis is

$$
V(2 n+1)=\prod_{j=1}^{n} \frac{\left(\begin{array}{c}
6 j-2 \\
2 j
\end{array}\right)}{2\left(\begin{array}{c}
4 j-1 \\
2 j
\end{array}\right)}
$$

A more refined conjecture is presented by Mills, Robbins, and Rumsey [50] relating this type of alternating sign matrices to the determinant $T_{n}(x, 1)$ in (3.10). Especially, 
$T_{n}(1,1)=\prod_{j=1}^{n} \frac{\left(\begin{array}{c}6 j-2 \\ 2 j\end{array}\right)}{2\left(\begin{array}{c}4 j-1 \\ 2 j\end{array}\right)}$ is conjectured to be the total number $V(2 n+1)$. As we saw in Section III, the same formula comes in as the special Hankel determinant $d_{n}^{(1)}$, where in (1.2) we choose generalized Catalan numbers $\frac{1}{3 m+1}\left(\begin{array}{c}3 m+1 \\ m\end{array}\right)$ as entries.

Let us consider this conjecture a little closer. If an alternating sign matrix (short: ASM) is invariant under a reflection about a vertical axis, it must obviously be of odd order $2 n+1$, since otherwise there would be a row containing two successive nonzero entries with the same sign. For the same reason, such a matrix cannot contain any 0 in its central column as seen in the example (4.1).

In [15], cf. also [16], Ch. 7.1, an equivalent counting problem via a bijection to families of disjoint paths in a square lattice is presented. Denote the vertices corresponding to the entry $a_{i j}$ in the ASM by $(i, j), i, j=0, \ldots, n-1$. Then following the outermost path from $(n-1,0)$ to $(0, n-1)$, the outermost path in the remaining graph from $(0, n-2)$ to $(n-2,0)$, and so on until the path from $(0,1)$ to $(1,0)$ one obtains a collection of lattice paths, which are edge-disjoint but may share vertices.

Since there can be no entry 0 in the central column of the ASM invariant under a reflection about a vertical axis, the entries $a_{0, n}, a_{2, n}, a_{4, n}, \ldots, a_{2 n, n}$ must be 1 and $a_{1, n}=a_{3, n}=a_{5, n}=$ $\ldots a_{2 n, n}=-1$. This means that for $i=0, \ldots n-1$ the path from $(2 n-i, 0)$ to $(0,2 n-i)$ must go through $(2 n-i, n)$ where it changes direction from East to North and after that in $(2 n-i-1, n)$ it again changes direction to East and continues in $(2 n-i-1, n+1)$. Because of the reflection-invariance about the central column the matrix of size $(2 n+$ $1) \times(2 n+1)$ is determined by its column numbers. $n+1, n+2, \ldots 2 n$. So, by the above considerations the matrix can be reconstructed from the collection of subpaths $\left(\mu_{0}, \mu_{1}, \ldots, \mu_{n-1}\right)$ where $\mu_{i}$ leads from $(2 n-i-1, n+1)$ to $(0,2 n-i)$.

By a reflection about the horizontal and a 90 degree turn to the left, we now map the collection of these paths to a collection of paths $\left(\nu_{0}, \nu_{1}, \ldots, \nu_{n-1}\right)$ the integer lattice $\mathbb{Z} \times \mathbb{Z}$, such that the inner most subpath in the collection leads from $(-1,0)$ to $(0,0)$ and path $\nu_{i}$ leads from $(-2 i-1,0)$ to $(0, i)$.

Denoting by $v_{i, s}$ the $y$-coordinate of the $s$-th vertical step (where the path is followed from the right to the left) in path number $i, i=1, \ldots, n-1$ - path $\nu_{0}$ does not contain vertical steps - the collection of paths $\left(\nu_{0}, \nu_{1}, \ldots, \nu_{n-1}\right)$ can be represented by a two-dimensional array (plane partition) of positive integers

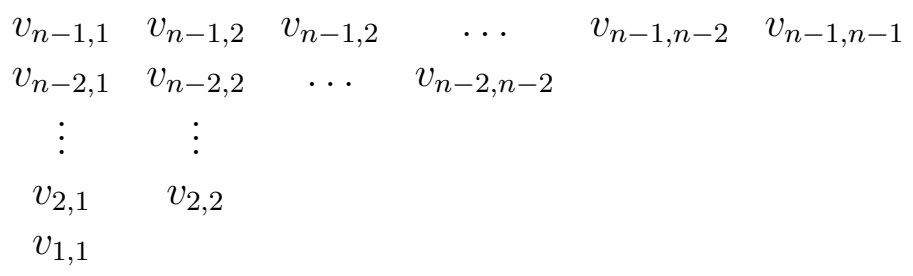

with weakly decreasing rows, i. e. $v_{i, 1} \geq v_{i, 2} \geq \ldots \geq v_{i, i}$ for all $i$, and the following restrictions:

1) $2 i-1 \leq v_{i, 1} \leq 2 i+1$ for all $i=1, \ldots, n-1$,

2) $v_{i, s}-v_{i, s-1} \leq 1$ for all $i, s$ with $s>i$. 
3) $v_{i+1, i+1} \geq v_{i, i}$ for all $1 \leq i \leq n-1$.

So for $n=1$ there is only the empty array and for $n=2$ there are the three possibilities $v_{1,1}=1, v_{1,1}=2$, or $v_{1,1}=3$. For $n=3$ the following 26 arrays obeying the above restrictions exist:

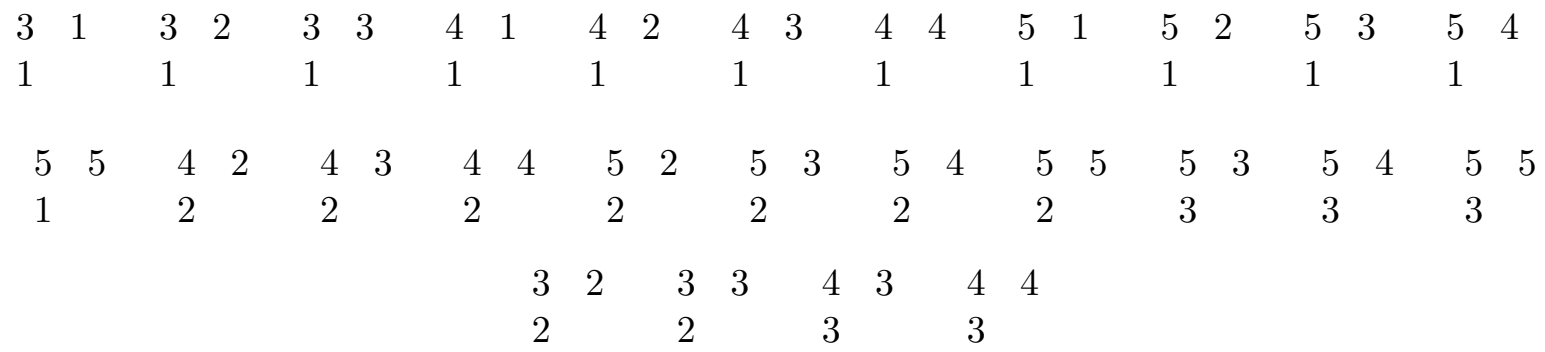

Now consider a collection $\left(\gamma_{0}, \gamma_{1}, \ldots, \gamma_{n-1}\right)$ of vertex disjoint paths in the integer lattice as required in Theorem 3.1, where the single paths are not allowed to cross the diagonal $2 x=y$ and path $\gamma_{i}$ leads from $(-i,-2 i)$ to $(i+1,2 i+2)$. Obviously, the initial segment of path $\gamma_{i}$ must be the line connecting $(-i,-2 i)$ and $(-i, i+2)$. Since no variation is possible in this part, we can remove these initial segments and obtain a collection $\left(\eta_{0}, \ldots, \eta_{n-1}\right)$ of vertex-disjoint paths, where now $\eta_{i}$ leads from $(-i, i+2)$ to $(i+1,2 i+2)$.

We now denote by $v_{i, s}$ the position of the $s$-th vertical step (i. e. the number of the horizontal step before the $s$-th vertical step in the path counted from right to left) in path $\eta_{i}, i=1, \ldots, n-1$ and obtain as a representation of the collection $\left(\eta_{0}, \ldots, \eta_{n-1}\right)$ a two-dimensional array of positive integers with weakly decreasing rows as in (4.3), where the restrictions now are:

1) $2 i-1 \leq v_{i, 1} \leq 2 i+1$ for all $i=1, \ldots, n$,

2') $v_{i, s}-v_{i, s-1} \leq 2$ for all $i, s$ with $s>i$.

Again, for $n=1$ there is only the empty array and for $n=2$ there are the three choices $v_{1,1}=1, v_{1,1}=2$, or $v_{1,1}=3$ as above. For $n=3$ the first 22 arrays above also fulfill the conditions 2 '), whereas the four arrays in the last row do not. However, they can be replaced by

$\begin{array}{llllllll}4 & 1 & 5 & 1 & 5 & 1 & 5 & 2 \\ 2 & & 2 & & 3 & & 3\end{array}$

in order to obtain a total number of 26 as above. Unfortunately, we did not find a bijection between these two types of arrays or the corresponding collections of paths yet.

\section{Catalan - like Numbers and the Berlekamp - Massey Algorithm}

In this section we shall study two - dimensional arrays $l(m, j), m, j=0,1,2, \ldots$ and the matrices $L_{n}=(l(m, j))_{m, j=0,1, \ldots, n-1}$ defined by

$$
l(m, j)=T\left(x^{m} \cdot t_{j}(x)\right),
$$


where $T$ is the linear operator defined under (1.9). Application of the three-termrecurrence (1.19)

$$
t_{j}(x)=\left(x-\alpha_{j}\right) t_{j-1}(x)-\beta_{j-1} t_{j-2}(x)
$$

and the linearity of $T$ gives the recursion

$$
l(m, j)=l(m-1, j+1)+\alpha_{j+1} l(m-1, j)+\beta_{j} l(m-1, j-1)
$$

with initial values $l(m, 0)=c_{m}, l(0, j)=0$ for $j \neq 0$ (and $\beta_{0}=0$, of course). Especially, cf. also [78], p. 195,

$$
l(m, m)=c_{0} \beta_{1} \beta_{2} \cdots \beta_{m}, \quad l(m+1, m)=c_{0} \beta_{1} \beta_{2} \cdots \beta_{m}\left(\alpha_{1}+\alpha_{2}+\ldots+\alpha_{m+1}\right)
$$

We shall point out two connections of the matrices $L_{n}$ to Combinatorics and Coding Theory. Namely, for the case that $\beta_{j}=1$ for all $j$ the matrices $L_{n}$ occur in the derivation of Catalan - like numbers as defined by Aigner in [2]. They also can be determined in order to find the factorization $L_{n}=A_{n} \cdot U_{n}^{t}$, where $A_{n}$ is a nonsingular Hankel matrix of the form (1.1) and $U_{n}$ is the matrix (1.12) with the coefficients of the orthogonal polynomials in (1.8). Via formula (5.3) the Berlekamp - Massey algorithm can be applied to find the parameters $\alpha_{j}$ and $\beta_{j}$ in the three - term recurrence of the orthogonal polynomials (1.8).

Aigner in [2] introduced Catalan - like numbers and considered Hankel determinants consisting of these numbers. For positive reals $a, s_{1}, s_{2}, s_{3}, \ldots$ Catalan - like numbers $C_{m}^{(a, \vec{s})}, \vec{s}=\left(s_{1}, s_{2}, s_{3}, \ldots\right)$ can be defined as entries $b(m, 0)$ in a two - dimensional array $b(m, j), m=0,1,2, \ldots, j=0,1, \ldots, m$, with initial conditions $b(m, m)=1$ for all $m=$ $0,1,2, \ldots, b(0, j)=0$ for $j>0$, and recursion

$$
\begin{gathered}
b(m, 0)=a \cdot b(m-1,0)+b(m-1,1), \\
b(m, j)=b(m-1, j-1)+s_{j} \cdot b(m-1, j)+b(m-1, j+1) \text { for } j=1, \ldots, m .
\end{gathered}
$$

The matrices $B_{n}=(b(m, j))_{m, j=0, \ldots, n-1}$, obtained from this array, have the property that $B_{n} \cdot B_{n}^{t}$ is a Hankel matrix, which has, of course, determinant 1, see also [66] for the Catalan numbers.

The matrices $B_{n}$ can be generalized in several ways. For instance, with $\beta_{j}=1$ for all $j \geq 2, \alpha_{1}=a$ and $\alpha_{j+1}=s_{j}$ for $j \geq 2$ the recursion (5.2) now yields the matrix $L_{n}=\left(l(m, j)_{m, j=0, \ldots, n-1}\right)$. Another generalization of the matrices $B_{n}$ will be mentioned below.

Aigner [2] was especially interested in Catalan - like numbers with $s_{j}=s$ for all $j$ and some fixed $s$ denoted here by $C_{m}^{(a, s)}$. In the example below the binomial coefficients $\left(\begin{array}{c}2 m+1 \\ m\end{array}\right)$ arise as $C_{m}^{(3,2)}$. 


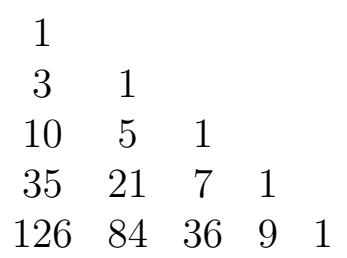

So, by the previous considerations, choosing $c_{m}=C_{m}^{(a, \vec{s})}$ we have that the determinant $d_{n}^{(0)}=1$ for all $n$. In [2] it is also computed the determinant $d_{n}^{(1)}$ via the recurrence

$$
d_{n}^{(1)}=s_{n-1} \cdot d_{n-1}^{(1)}-d_{n-2}^{(1)} .
$$

with initial values $d_{0}^{(1)}=1, d_{1}^{(1)}=a$.

\section{Remarks:}

1) One might introduce a new leading element $c_{-1}$ to the sequence $c_{0}, c_{1}, c_{2}, \ldots$ and define the $n \times n$ Hankel matrix $A_{n}^{(-1)}$ and its determinant $d_{n}^{(-1)}$ for this new sequence. Let $\left(c_{m}=C_{m}^{(s, s)}\right)_{m=0,1, \ldots}$ be the sequence of Catalan-like numbers with parameters $(s, s)$, $s>1$ and let $c_{-1}=1$. Let $A_{n}^{(k)}$ be the Hankel matrix of size $n \times n$ as under (1.2) and let $d_{n}^{(k)}$ denote its determinant. Then

$$
d_{n}^{(-1)}=(s-1)(n-1)+1, \quad d_{n}^{(0)}=1, \quad d_{n}^{(1)}=s n+1, \quad d_{n}^{(2)}=\sum_{j=1}^{n+1}(s j+1)^{2} .
$$

This result follows, since $d_{n}^{(0)}$ and $d_{n}^{(1)}$ are known from Propositions 6 and 7 in [2]. So the sequences $d_{n}^{(k)}$ are known for two successive $k$ 's, such that the formulae for $d_{n}^{(-1)}$ and $d_{n}^{(2)}$ are easily found using recursion (2.3).

2) In [2] it is shown that $C_{m}^{(1,1)}$ are the Motzkin numbers, $C_{m}^{(2,2)}$ are the Catalan numbers and $C_{m}^{(3,3)}$ are restricted hexagonal numbers. Guy [41] gave an interpretation of the numbers $C_{m}^{(4,4)}$ starting with $1,4,17,76,354, \ldots$. They come into play when determining the number of walks in the three - dimensional integer lattice from $(0,0,0)$ to $(i, j, k)$ terminating at height $k$, which never go below the $(i, j)$-plane. With the results of [2] their generating function is $\frac{1-4 x-\sqrt{1-8 x+12 x^{2}}}{2 x^{2}}$.

Lower triangular matrices $L_{n}$ as defined by (5.1) are also closely related to the Lanczos algorithm. Observe that with (5.3) we obtain the parameters in the three - term recursion in a form which was already known to Chebyshev in his algorithm in [19], p. 482, namely

$$
\alpha_{1}=\frac{l(1,0)}{l(0,0)} \text { and } \alpha_{j+1}=\frac{l(j+1, j)}{l(j, j)}-\frac{l(j, j-1)}{l(j-1, j-1)}, \quad \beta_{j}=\frac{l(j, j)}{l(j-1, j-1)} \text { for } j \geq 1
$$


Since further $l(m, 0)=c_{m}$ for all $m \geq 0$ by (5.3) it is $l(m-1,1)=l(m, 0)-\alpha_{1} l(m-1,0)$ and

$$
l(m-1, j+1)=l(m, j)-\alpha_{j+1} l(m-1, j)-\beta_{j} l(m-1, j-1)
$$

for $j>0$, from which the following recursive algorithm is immediate.

Starting with $\vec{l}_{1}=\left(\begin{array}{c}c_{0} \\ c_{1} \\ \vdots \\ c_{2 n-2}\end{array}\right)$ and defining $Z=\left(\begin{array}{ccccc}0 & 0 & \ldots & 0 & 0 \\ 1 & 0 & \ldots & 0 & 0 \\ 0 & 1 & \ldots & 0 & 0 \\ \vdots & \vdots & & \vdots & \vdots \\ 0 & 0 & \ldots & 1 & 0\end{array}\right)$ of size $(2 n-1) \times$ $(2 n-1)$ and $Z^{t}$ its transpose, we obtain recursively

$$
\overrightarrow{l_{1}}=Z^{t} \cdot \overrightarrow{l_{0}}-\alpha_{1} \vec{l}_{0}, \quad \vec{l}_{j+1}=Z^{t} \cdot \vec{l}_{j}-\alpha_{j+1} \cdot \vec{l}_{j}-\beta_{j} \cdot \vec{l}_{j-1} \quad \text { for } j>0
$$

The subvectors of the initial $n$ elements of $\vec{l}_{j+1}$ then form the $(j+1)$-th column $(j=$ $1, \ldots, n-2)$ of $L_{n}$.

In a similar way the matrix $U_{n}^{t}$, the transpose of the matrix (1.12) consisting of the coefficients of the orthogonal polynomials, can be constructed. Here $\vec{u}_{0}=\left(\begin{array}{c}1 \\ 0 \\ \vdots \\ 0\end{array}\right)$ is the first unit column vector of size $2 n-1$ and then the further columns are obtained via

$$
\vec{u}_{1}=Z \cdot \vec{u}_{0}-\alpha_{1} \cdot \vec{u}_{0}, \quad \vec{u}_{j+1}=Z \cdot \vec{u}_{j}-\alpha_{j+1} \cdot \vec{u}_{j}-\beta_{j} \cdot \vec{u}_{j-1}
$$

Again the first $n$ elements of $\vec{u}_{j}$ form the $j$-th column of $U_{n}^{t}$.

This is the asymmetric Lanczos algorithm yielding the factorization $A_{n} \cdot U_{n}^{t}=L_{n}$ as studied by Boley, Lee, and Luk [13], where $A_{n}$ is an $n \times n$ Hankel matrix as in (1.1). Their work is based on a former paper by Phillips [58]. The algorithm is $O\left(n^{2}\right)$ due to the fact that the columns in $L_{n}$ and $U_{n}^{t}$ are obtained only using the entries in the previous two columns.

The symmetric Lanczos algorithm in [13] yields the factorization $A_{n}=M_{n} \cdot D_{n} \cdot M_{n}^{t}$. Here, cf. [13], p. 120, $L_{n}=M_{n} \cdot D_{n}$ where $M_{n}=U_{n}^{-1}$ is the inverse of $U_{n}$ and $D_{n}$ is the diagonal matrix with the eigenvalues of $A_{n}$. A combinatorial interpretation of the matrix $M_{n}$ was given by Viennot [76].

When $D_{n}$ is the identity matrix, then $L_{n}=M_{n}$ and the matrix $M_{n}$ was used in [54] to derive combinatorial identities as for Catalan - like numbers. Namely, in [54], the Stieltjes matrix $S_{n}=M_{n}^{-1} \cdot \bar{M}_{n}$ was applied, where $\bar{M}_{n}=\left(m_{n+1, j}\right)_{m, j=0, \ldots, n-1}$ for $M_{n}=$ $\left(m_{n, j}\right)_{m, j=0, \ldots, n-1}$. Then 


$$
S_{n}=\left(\begin{array}{cccccc}
\alpha_{0} & 1 & 0 & 0 & \ldots & 0 \\
\beta_{0} & \alpha_{1} & 1 & 0 & \ldots & 0 \\
0 & \beta_{1} & \alpha_{2} & 1 & \ldots & 0 \\
\vdots & \vdots & \vdots & \vdots & & \vdots \\
0 & 0 & 0 & 0 & \ldots & \alpha_{n-1}
\end{array}\right)
$$

is tridiagonal with the parameters of the three - term recurrence on the diagonals.

Important for the decoding of $\mathrm{BCH}$ codes, studied in the following, is also a decomposition of the Hankel matrix $A_{n}=V_{n} D_{n} V_{n}^{t}$ as a product of a Vandermonde matrix $V_{n}$, its transpose $V_{n}^{t}$ and the diagonal matrix $D_{n}$. Here the parameters in the Vandermonde matrix are essentially the roots of the polynomial $t_{n}(x)$. This decomposition was known already to Baron Gaspard Riche de Prony [60] (rather known as the leading engineer in the construction of the Pont de la Concorde in Paris and as project head of the group producing the logarithmic and trigonometric tables from 1792 - 1801), cf. also [14].

Let us now discuss the relation of the Berlekamp - Massey algorithm to orthogonal polynomials. Via (5.3) the parameters $r_{j}$ in the Berlekamp - Massey algorithm presented below will be explained in terms of the three - term recurrence of the orthogonal polynomials related to $A_{n}$.

Peterson [56] and Gorenstein and Zierler [38] presented an algorithm for the decoding of $\mathrm{BCH}$ codes. The most time-consuming task is the inversion of a Hankel matrix $A_{n}$ as in (1.1), in which the entries $c_{i}$ now are syndromes resulting after the transmission of a codeword over a noisy channel. Matrix inversion, which takes $O\left(n^{3}\right)$ steps was proposed to solve equation (1.7).

Berlekamp found a way to determine the $a_{n, j}$ in (1.7) in $O\left(n^{2}\right)$ steps. His approach was to determine them as coefficients of a polynomial $u(x)$ which is found as appropriate solution of the "key equation"

$$
F(x) u(x)=q(x) \bmod x^{2 t+1} .
$$

Here the coefficients $c_{0}, \ldots, c_{2 t}$ up to degree $2 t$ of $F(x)$ can be calculated from the received word. Further, the roots of $u(x)$ yield the locations of the errors (and also determine $q(x)$ ). By the application in Coding Theory one is interested in finding polynomials of minimum possible degree fulfilling the key equation. This key equation is solved by iteratively calculating solutions $\left(q_{k}(x), u_{k}(x)\right)$ to $F(x) u_{k}(x)=q_{k}(x) \bmod z^{k+1}, k=0, \ldots, 2 t$.

Massey [48] gave a variation of Berlekamp's algorithm in terms of a linear feedback shift register. The algorithm is presented by Berlekamp in [9]. We follow here Blahut's book [11], p. 180.

The algorithm consist in constructing a sequence of shift registers $\left(\ell_{j}, u_{j}(x)\right), j=1, \ldots$, $2 n-2$, where $\ell_{j}$ denotes the length (the degree of $u_{j}$ ) and

$$
u_{j}(x)=b_{j, j} x^{j}+b_{j, j-1} x^{j-1}+\ldots+b_{j, 1} x+1 .
$$


the feedback-connection polynomial of the $j$-th shift register. For an introduction to shift registers see, e.g., [11], pp. 131, The Berlekamp - Massey algorithm works over any field and will iteratively compute the polynomials $u_{j}(x)$ as follows using a second sequence of polynomials $v_{j}(x)$.

Berlekamp - Massey Algorithm (as in [11], p. 180): Let $u_{0}(x)=1, v_{0}(x)=1$ and $\ell_{0}=0$. Then for $j=1, \ldots, 2 n-2$ set

$$
\begin{gathered}
r_{j}=\sum_{t=0}^{\ell_{j}} b_{j-1, t} c_{j-1-t}, \\
\ell_{j}=\delta_{j}\left(j-\ell_{j-1}\right)+\left(1-\delta_{j}\right) \ell_{j-1}, \\
\left(\begin{array}{l}
u_{j}(x) \\
v_{j}(x)
\end{array}\right)=\left(\begin{array}{cc}
1 & -r_{j} x \\
\delta_{j} \cdot 1 / r_{j} & \left(1-\delta_{j}\right) x
\end{array}\right) \cdot\left(\begin{array}{l}
u_{j-1}(x) \\
v_{j-1}(x)
\end{array}\right),
\end{gathered}
$$

where

$$
\delta_{j}=\left\{\begin{array}{cr}
1 & \text { if } r_{j} \neq 0 \text { and } 2 \ell_{j-1} \leq j-1 \\
0 & \text { otherwise }
\end{array} .\right.
$$

Goppa [33] introduced a more general class of codes (containing the $\mathrm{BCH}$ - codes as special case) for which decoding is based on the solution of the key equation $F(x) u(x)=q(x)$ mod $G(x)$ for some polynomial $G(x)$. Berlekamp's iterative algorithm does not work for arbitrary polynomial $G(x)$ (cf. [10]). Sugiyama et al. [73] suggested to solve this new key equation by application of the Euclidean algorithm for the determination of the greatest common divisor of $F(x)$ and $G(x)$, where the algorithm stops, when the polynomials $u(x)$ and $q(x)$ of appropriate degree are found. They also showed that for BCH codes the Berlekamp algorithm usually has a better performance than the Euclidean algorithm. A decoding procedure based on continued fractions for separable Goppa codes was presented by Goppa in [34] and later for general Goppa codes in [35]. The relation of Berlekamp's algorithm to continued fraction techniques was pointed out by Mills [49] and thoroughly studied by Welch and Scholtz [79].

Cheng [20] analysed that the sequence $\ell_{j}$ provides the information when Berlekamp's algorithm completes one iterative step of the continued fraction, which happens when $\ell_{j}<j+\frac{1}{2}$ and when $\ell_{j} \neq \ell_{j+1}$. This means that if this latter condition is fulfilled, the polynomials $q_{j}(x)$ and $u_{j}(x)$ computed so far give the approximation $\frac{q_{j}(x)}{u_{j}(x)}$ to $F(x)$, which would also be obtained as convergent from the continued fractions expansion of $F(x)$.

Indeed, the speed of the Berlekamp - Massey algorithm is due to the fact that it constructs the polynomials $u_{j}(x)$ in the denominator of the convergent to $F(x)$ via the three - term recursion

$$
u_{j}(x)=u_{j-1}(x)-\frac{r_{j}}{r_{m}} x^{j-m} u_{m-1}(x)
$$


Here $r_{m}$ and $r_{j}$ are different from 0 and $r_{m+1}=\ldots=r_{j-1}=0$, which means that in (5.7) $\delta_{m+1}=\ldots=\delta_{j-1}=0$ and $\delta_{j}=1$, such that at time $j$ for the first time after $m$ a new shift register must be designed. This fact can be proved inductively as in [12], p. 374 . An approach reflecting the mathematical background of these "jumps" via the Iohvidov index of the Hankel matrix or the block structure of the Padé table is carried out by Jonckheere and Ma [44].

Several authors (e.g. [45], p. 156, [43], [44], [13]) point out that the proof of the above recurrence is quite complicated or that there is need for a transparent explanation. We shall see now that the analysis is much simpler for the case that all principle submatrices of the Hankel matrix $A_{n}$ are nonsingular. As a useful application, then the $r_{j}$ 's yield the parameters from the three - term recurrence of the underlying polynomials. Via (5.5) the three - term recurrence can also be transferred to the case that calculations are carried out over finite fields.

So, let us assume from now on that all principal submatrices $A_{i}, i \leq n$ of the Hankel matrix $A_{n}$ are nonsingular. For this case, Imamura and Yoshida [43] demonstrated that $\ell_{j}=\ell_{j-1}=\frac{j}{2}$ for even $j$ and $\ell_{j}=j-\ell_{j-1}=\frac{j+1}{2}$ for odd $j$ such that $\delta_{j}$ is 1 if $j$ is odd and 0 if $j$ is even $\left(\frac{q_{2 j}(x)}{u_{2 j}(x)}\right.$ then are the convergents to $\left.F(x)\right)$.

This means that there are only two possible recursions for $u_{j}(x)$ depending on the parity of $j$, namely

$$
u_{2 j}(x)=u_{2 j-1}(x)-\frac{r_{2 j}}{r_{2 j-1}} x u_{2 j-2}(x), \quad u_{2 j-1}(x)=u_{2 j-2}(x)-\frac{r_{2 j-1}}{r_{2 j-3}} x^{2} u_{2 j-4}(x) .
$$

So the algorithm is simplified in (5.7) and we obtain the recursion

$$
\left(\begin{array}{l}
u_{2 j}(x) \\
v_{2 j}(x)
\end{array}\right)=\left(\begin{array}{cc}
1-\frac{r_{2 j}}{r_{2 j-1}} x & -r_{2 j-1} x \\
\frac{1}{r_{2 j-1}} x & 0
\end{array}\right) \cdot\left(\begin{array}{c}
u_{2 j-2}(x) \\
v_{2 j-2}(x)
\end{array}\right) .
$$

By the above considerations we have the following three-term recurrence for $u_{2 j}(x)$ (and also for $q_{2 j}(x)$ with different initial values).

$$
u_{2 j}(x)=\left(1-\frac{r_{2 j}}{r_{2 j-1}} x\right) u_{2 j-2}(x)-\frac{r_{2 j-1}}{r_{2 j-3}} x^{2} u_{2 j-4}(x) .
$$

Since the Berlekamp - Massey algorithm determines the solution of equation (1.9) it must be

$$
x^{j} \cdot u_{2 j}\left(\frac{1}{x}\right)=t_{j}(x) .
$$

as under (1.8). This is consistent with (1.16) where we consider the function $F\left(\frac{1}{x}\right)$ rather than $F(x)$. By the previous considerations, for $t_{j}(x)$, we have the recurrence

$$
t_{j}(x)=\left(x-\frac{r_{2 j}}{r_{2 j-1}}\right) t_{j-1}(x)-\frac{r_{2 j-1}}{r_{2 j-3}} t_{j-2}(x)
$$


Equation (5.11) now allows us to give a simple interpretation of the calculations in the single steps carried out in the course of the Berlekamp - Massey algorithm for the special case that all principle submatrices of the Hankel matrix $A_{n}$ are nonsingular.

Proposition 5.1: Let $A_{n}$ be a Hankel matrix with real entries such that all principal submatrices $A_{i}, i=1, \ldots, n$ are nonsingular and let $T$ be the linear operator mapping $T\left(x^{l}\right)=c_{l}$ as in (1.9). Then for the parameters $r_{j}$ obtained via (5.6) it is

$$
\begin{aligned}
& r_{2 j-1}=T\left(x^{j-1} \cdot t_{j-1}(x)\right)=c_{0} \beta_{1} \beta_{2} \cdots \beta_{j-1}, \\
& r_{2 j}=\alpha_{j} T\left(x^{j-1} \cdot t_{j-1}(x)\right)=c_{0} \beta_{1} \beta_{2} \cdots \beta_{j-1} \alpha_{j},
\end{aligned}
$$

where $\alpha_{j}$ and $\beta_{1}, \ldots, \beta_{j-1}$ are the parameters from the three-term recurrence of the orthogonal polynomials $t_{i}(x), i=0, \ldots, j$.

Proof: The proposition, of course, follows directly from (5.11), since the three - term recurrence immediately yields the formula for the $r_{j}$ 's. Let us also verify the identities directly. From the considerations under (5.6) to (5.11) it is clear that the degree of $u_{2 j-2}$ is $j-1$. Hence in this case $b_{2 j-2, j}=b_{2 j-2, j+1}=\ldots=b_{2 j-2,2 j-2}=0$ in (5.6) and

$$
\begin{gathered}
r_{2 j-1}=\sum_{t=0}^{j-1} b_{2 j-2, t} c_{2 j-2-t}=\sum_{t=0}^{j-1} b_{2 j-2, t} T\left(x^{2 j-2-t}\right) \\
=T\left(\sum_{t=0}^{j-1} b_{2 j-2, t} x^{2 j-2-t}\right)=T\left(x^{j-1} \sum_{t=0}^{j-1} b_{2 j-2, t} x^{j-1-t}\right)=T\left(x^{j-1} \sum_{t=0}^{j-1} b_{2 j-2, j-1-t} x^{t}\right) \\
=T\left(x^{j-1} \sum_{t=0}^{j-1} a_{j-1, t} x^{t}\right)=T\left(x^{j-1} t_{j-1}(x)\right)=c_{0} \beta_{1} \beta_{2} \cdots \beta_{j-1}
\end{gathered}
$$

where the last equation follows by (5.3). A similar calculation shows that

$$
r_{2 j}=T\left(x^{j} t_{j-1}(x)-\frac{r_{2 j-1}}{r_{2 j-3}} x^{j-1} t_{j-2}(x)\right)=T\left(x^{j} t_{j-1}(x)-\beta_{j-1} x^{j-1} t_{j-2}(x)\right)
$$

since by the previous calculation $\frac{r_{2 j-1}}{r_{2 j-3}}=\beta_{j-1}$. So by (5.3) further

$$
r_{2 j}=c_{0} \beta_{1} \beta_{2} \cdots \beta_{j-1}\left[\left(\alpha_{1}+\alpha_{2}+\ldots+\alpha_{j}\right)-\left(\alpha_{1}+\alpha_{2}+\ldots+\alpha_{j-1}\right)\right]=c_{0} \beta_{1} \beta_{2} \cdots \beta_{j-1} \alpha_{j} .
$$

\section{Remarks:}

1) Observe that with Proposition 5.1, the Berlekamp - Massey algorithm can be applied to determine the coefficients $\alpha_{j}$ and $\beta_{j}$ from the three - term recurrence of the orthogonal polynomials $t_{j}(x)$. From the parameters $r_{2 j-1}$ obtained by (5.6) in the odd steps of the iteration $\beta_{j-1}=\frac{r_{2 j-1}}{r_{2 j-3}}$ can be immediately calculated, and in the even steps $\alpha_{j}=$ 
$\frac{r_{2 j}}{r_{2 j-1}}$ is obtained. By (1.15) and (1.20) it is $\beta_{j-1}=\frac{r_{2 j-1}}{r_{2 j-3}}=\frac{\operatorname{det}\left(A_{j}\right) \operatorname{det}\left(A_{j-2}\right)}{\operatorname{det}\left(A_{j-1}\right)^{2}}$. Hence $r_{2 j-1}=\frac{\operatorname{det}\left(A_{j}\right)}{\operatorname{det}\left(A_{j-1}\right)}$, which means that the Berlekamp - Massey algorithm also yields a fast procedure to compute the determinant of a Hankel matrix.

2) By Proposition 5.1 the identity (5.6) reduces to

$$
\sum_{t=0}^{j} a_{j, t} c_{j+t}=c_{0} \beta_{1} \beta_{2} \cdots \beta_{j}
$$

where the $a_{j, t}$ are the coefficients of the polynomial $t_{j}(x)$, the $\beta_{i}$ 's are the coefficients in their three - term recurrence and the $c_{i}$ 's are the corresponding moments. For the classical orthogonal polynomials all these parameters are usually known, such that one might also use (5.6) in the Berlekamp - Massey algorithm to derive combinatorial identities.

\section{References}

[1] M. Aigner, "Motzkin numbers", Europ. J. Combinatorics 19, 1998, 663 - 675.

[2] M. Aigner, "Catalan-like numbers and determinants", J. Combin. Theory Ser. A 87, $1999,33-51$.

[3] M. Aigner, "A characterization of the Bell numbers", Discrete Math. 205, no. 1-3, 1999, 207-210.

[4] G. E. Andrews, "Plane partitions (III): The weak Macdonald conjecture", Invent. Math. 53, 1979, 193 - 225.

[5] G. E. Andrews, "Pfaff's method. I. The Mills-Robbins-Rumsey determinant", Discrete Math. 193, no. 1-3, 1998, 43-60.

[6] G. E. Andrews and D. Stanton, "Determinants in plane partition enumeration", Europ. J. Combin. 19, 1998, no. 3, $273-282$.

[7] R. Askey and M. Ismail, Recurrence Relations, Continued Fractions and Orthogonal Polynomials, Mem. Am. Math. Soc. 300, 1984.

[8] E. A. Bender and D. E. Knuth, "Enumeration of plane partitions", J. Combin. Theory Ser. A 13, 1972, $40-54$.

[9] E. R. Berlekamp, Algebraic Coding Theory, McGraw-Hill, 1968.

[10] E. R. Berlekamp, "Goppa Codes", IEEE Trans. Inform. Theory 19, 1973, 590 - 592.

[11] R. E. Blahut, Theory and Practice of Error Control Codes, Addison - Wesley, 1984.

[12] R. E. Blahut, Fast Algorithms for Digital Signal Processing, Addison - Wesley, 1985. 
[13] D. L. Boley, T. J. Lee, and F. T. Luk, "The Lanczos algorithm and Hankel matrix factorization", Lin. Alg. Appl. 172, 1992, 109 - 133.

[14] D. L. Boley, F. T. Luk, and D. Vandevoorde, "A fast method to diagonalize a Hankel matrix", Lin. Alg. Appl. 284, 1998, $41-52$.

[15] M. Bousquet - Mélou and L. Habsieger, "Sur les matrices à signes alternants", Discrete Math. 139, 1995, $57-72$.

[16] D. M. Bressoud, Proofs and Confirmations, Cambridge Univ. Press, 1999.

[17] C. Brezinski, Padé-Type Approximation and General Orthogonal Polynomials, Birkhäuser, 1980.

[18] Lewis Carroll, Alice's Adventures in Wonderland, 1865.

[19] P. L. Chebyshev, "Sur l'interpolation par la méthode des moindres carrés", Mém. Acad. Impér. Sci. St. Pétersbourg (7) 1 (15), 1859, 1 - 24, also: Oeuvres I, 473 - 489.

[20] U. Cheng, "On the continued fraction and Berlekamp's algorithm", IEEE Trans. Inform. Theory 30, 1984, $541-544$.

[21] S. H. Choi and D. Gouyou - Beauchamps, "Enumeration of generalized Young tableaux with bounded height", Theoret. Comp. Sci. 117, 1993, $137-151$.

[22] W. Chu, "Binomial convolutions and determinant identities", Discrete Math. 204, 1999, $129-153$.

[23] P. Delsarte, "Nombres de Bell et polynômes de Charlier", C. R. Acad. Sci. Paris (series A), t. 287, 1978, $271-273$.

[24] M. Desainte-Catherine and X. G. Viennot, "Enumeration of certain Young tableaux with bounded height", Combinatoire Énumérative (Montreal 1985), Lect. Notes Math. 1234, 1986, $58-67$.

[25] C. L. Dodgson, "Condensation of determinants", Proceedings of the Royal Society of London 15, 1866, 150 - 155.

[26] P. Flajolet, "Combinatorial aspects of continued fractions", Discrete Math. 32, 1980, $125-161$.

[27] P. Flajolet, "On congruences and continued fractions for some classical combinatorial quantities", Discrete Math. 41, 1982, 145 - 153.

[28] D. Foata, "Combinatoire des identités sur les polynômes orthogonaux", Proc. International Congress of Mathematicians, Warsaw, 19831541 - 1553.

[29] I. Gessel, "A probabilistic method for lattice path enumeration", J. Statist. Plann. Inference 14, 1986, $49-58$. 
[30] I. Gessel and R. Stanley, "Algebraic enumeration". in R. L. Graham, M. Grötschel, and L. Lovasz, Handbook of Combinatorics, Vol. 2, Wiley, 1996, 1021 - 1069,

[31] I. Gessel and X. G. Viennot, "Binomial determinants, paths and hook length formulae", Advances in Mathematics 58, 1985, 300 - 321.

[32] I. Gessel and X. G. Viennot, Determinants, Paths, and Plane Partitions, Preprint, 1989.

[33] V. D. Goppa, "A new class of linear correcting codes", Problemy Peredachi Informatsii 6, no. 3, 1970, 24 - 30 (in Russian).

[34] V. D. Goppa, "Rational representation of codes and (L,g) codes", Problemy Peredachi Informatsii 7, no. 3, 1971, 41 - 49 (in Russian).

[35] V. D. Goppa, "Decoding and diophantine approximations", Problems of Control and Information Theory 5, no. 3, 1975, 195-206.

[36] B. Gordon, "A proof of the Bender - Knuth conjecture", Pacific J. Math. 108, 1983, $99-113$.

[37] D. C. Gorenstein, W. W. Peterson, and N. Zierler, "Two-error correcting BoseChaudhuri codes are quasi-perfect", Information and Control 3, 1960, 291 - 294.

[38] D. C. Gorenstein and N. Zierler, "A class of error-correcting codes in $p^{m}$ symbols", J. Soc. Indus. Appl. Math. 9, 1961, $207-214$.

[39] R. L. Graham, D. E. Knuth, and O. Patashnik, Concrete Mathematics, Addison Wesley, 1988.

[40] A. J. Guttmann, A. L. Owczarek, and X. G. Viennot, "Vicious walkers and Young tableaux I: without walls", J. Phys. A: Math. Gen. 31, 1998, 8123 - 8135.

[41] R. K. Guy, "Catwalks, sandsteps and Pascal pyramids", J. Integer Sequences 3, 2000, Article 00.1.6.

[42] P. Hilton and J. Pedersen, "Catalan numbers, their generalization, and their uses", Math. Intelligencer 13, no. 2, 1991, $64-75$.

[43] K. Imamura and W. Yoshida, "A simple derivation of the Berlekamp - Massey algorithm and some applications", IEEE Trans. Inform. Theory 33, 1987, 146 - 150.

[44] E. Jonckheere and C. Ma, "A simple Hankel interpretation of the Berlekamp - Massey algorithm", Lin. Alg. Appl. 125, 1989, $65-76$.

[45] S. Lin and D. J. Costello, Error - Control Coding, Prentice - Hall, 1983.

[46] B. Lindström, "On the vector representation of induced matroids", Bull. London Math. Soc. 5, 1973, $85-90$. 
[47] M. E. Mays and J. Wojciechowski, "A determinant property of Catalan numbers", Discrete Math. 211, 2000, 125 - 133.

[48] J. L. Massey, "Shift register synthesis and BCH decoding", IEEE Trans. Inform. Theory 15, 1969, $122-127$.

[49] W. H. Mills, "Continued fractions and linear recurrences", Math. Comp. 29, no. 129, $1975,173-180$.

[50] W. H. Mills, D.P. Robbins, and H. Rumsey Jr., "Enumeration of a symmetry class of plane partitions", Discrete Math. 67, 1987, 43 - 55.

[51] S. G. Mohanty, Lattice path counting and applications, Academic Press, 1979.

[52] T. Muir, Theory of Determinants, Dover, 1960.

[53] T. V. Narayana, Lattice Path Combinatorics, University of Toronto Press, 1979.

[54] P. Peart and W.--J. Woan, "Generating functions via Hankel and Stieltjes matrices", J. Integer Sequences 3, 2000, Article 00.2.1.

[55] O. Perron, Die Lehre von den Kettenbrüchen, Chelsea Publishing Company, 1929.

[56] W. W. Peterson, "Encoding and error-correction procedures for the Bose-Chaudhuri codes", Trans. IRE 6, 1960, $459-470$.

[57] M. Petkovšek and H. S. Wilf, "A high-tech proof of the Mills-Robbins-Rumsey determinant formula", Electron. J. Combin. 3, no. 2, 1996, \#R19.

[58] J. L. Phillips, "The triangular decomposition of Hankel matrices", Math. Comp. 25 (115), 1971, $599-602$.

[59] G. Polya and G. Szegö, Aufgaben und Lehrsätze aus der Analysis, Vol. II, 3rd ed., 1964.

[60] G. de Prony, "Essai expérimental et analytique sur les lois de la dilatabilité de fluides élastiques et sur les celles de la force expansive de la vapeur de l' alcool, à différentes températures", J. de l'École Polytechnique 1, cahier 22, 1795, 24 - 76.

[61] C. Radoux, "Déterminants de Hankel et théorème de Sylvester", Proc. 28th Séminaire Lotharingien, 1992, $115-122$.

[62] C. Radoux, "Addition formulas for polynomials built on classical combinatorial sequences", J. Comp. Appl. Math. 115, 2000, $471-477$.

[63] J. Riordan, An Introduction to Combinatorial Analysis, Wiley, 1958.

[64] J. Riordan, Combinatorial Identities, Wiley, 1968. 
[65] H. Rutishauser, Der Quotienten-Differenzen-Algorithmus, Birkhäuser, 1957.

[66] L. W. Shapiro, "A Catalan triangle", Discrete Math., 14, 1976, 83 - 90.

[67] R. P. Stanley, "Theory and application of plane partitions", Studies in Applied Mathematics 50, 1971, Part 1: 167 - 189, Part 2: 259 - 279.

[68] R. P. Stanley, "A baker's dozen of conjectures concerning plane partitions", Combinatoire Énumérative (Montreal 1985), Lect. Notes Math. 1234, 1986, 285-293.

[69] R. P. Stanley, Enumerative Combinatorics 2, Cambridge, 1999.

[70] T. J. Stieltjes, "Recherches sur les fractions continues", Ann. Fac. Sci. Toulouse 8, 1894, J.1 - 122, 1895, A.1 - 47.

[71] T. J. Stieltjes, Oeuvres Complètes, Springer, 1993.

[72] V. Strehl, Contributions to the combinatorics of some families of orthogonal polynomials, mémoire, Erlangen, 1982.

[73] Y. Sugiyama, M. Kasahara, S. Hirawawa, and T. Namekawa, "A method for solving key equation for decoding Goppa codes", Information and Control 27, 1975, 87 - 99.

[74] U. Tamm, "Hankel matrices in coding theory and combinatorics", Preprint 00-055, SFB 343 "Diskrete Strukturen in der Mathematik", Univ. of Bielefeld, July 2000.

[75] U. Tamm, "Lattice paths not touching a given boundary", J. Statist. Planning and Inference, submitted, 2000.

[76] X. G. Viennot, "A combinatorial theory for general orthogonal polynomials with extensions and applications", Polynômes Orthogonaux et Applications, Proceedings, Bar - le - Duc, Springer 1984, 139 - 157.

[77] X. G. Viennot, A combinatorial interpretation of the quotient - difference algorithm, Preprint, 1986.

[78] H. S. Wall, Analytic Theory of Continued Fractions, Chelsea Publ. Company, 1948.

[79] L. R. Welch and R. A. Scholtz, "Continued fractions and Berlekamp's algorithm", IEEE Trans. Inform. Theory 25, 1979, $19-27$.

[80] D. Zeilberger, "Proof of the alternating sign matrix conjecture", Electron. J. Combin. 3, 1996 \#R13, also in: The Foata Festschrift, ed. J. Désarmémien, A. Kerber, and V. Strehl, 289 - 273, Gap, France.

[81] D. Zeilberger, "Proof of the refined alternating sign matrix conjecture", New York Journal of Mathematics 2, 59 - 68, 1996.

[82] D. Zeilberger, "Dodgson's determinant-evaluation rule proved by TWO-TIMING MEN and WOMEN", Electron. J. Combin. 4 (2), 1997, \#R22. 